\title{
Bottomonia suppression in heavy-ion collisions from anti-de Sitter space/conformal field theory
}

\author{
N. N. Barnard* and W. A. Horowitz \\ Department of Physics, University of Cape Town, Private Bag X3, Rondebosch 7701, South Africa
}

(Received 30 June 2017; revised manuscript received 31 July 2019; published 21 January 2020)

\begin{abstract}
We compute for the first time the suppression of bottomonia in a strongly coupled quark-gluon plasma (QGP) and compare the results to those from a weakly coupled QGP. Using imaginary time techniques we numerically determine the real and imaginary parts of the ground-state binding energy of the bottomonia in one potential computed from anti-de Sitter space/conformal field theory (AdS/CFT) and another computed from perturbative quantum chromodynamics. We confirm the strong-coupling binding energies by independently deriving the meson spectrum in AdS/CFT using semiclassical, rotating open strings. We then use these binding energies in a suppression model to determine the $\Upsilon(1 \mathrm{~S})$ nuclear modification factor in $\sqrt{s}_{N N}=2.76-\mathrm{TeV} \mathrm{Pb}+\mathrm{Pb}$ collisions. AdS/CFT significantly overpredicts the suppression compared to data, although the predictive power of our calculation is limited by its significant sensitivity to the exact details of the suppression model.
\end{abstract}

DOI: 10.1103/PhysRevC.101.014911

\section{INTRODUCTION}

There is abundant evidence that high-multiplicity collisions at the Relativistic Heavy Ion Collider (RHIC) and the Large Hadron Collider (LHC) yield qualitatively new physics never before seen at previous colliding energies [1-7]. A natural explanation for these novel observations is that in these high-multiplicity collisions a new phase of colored matter is created, known as the quark-gluon plasma (QGP), in which the relevant degrees of freedom for the strong nuclear force are no longer hadrons [8]. Currently, divergent ideas for the relevant dynamics of the QGP medium in high-multiplicity hadronic collisions have had success in describing various experimental observables. For example, assuming the medium is best described using the strong-coupling techniques of antide-Sitter space/conformal field theory (AdS/CFT) explains the success of the picture of a rapidly thermodynamizing, nearly inviscid fluid that couples strongly to high-transversemomentum (high- $p_{T}$ ) open partons [9-19]. Simultaneously, weak-coupling techniques from perturbative quantum chromodynamics (pQCD) have also shown success in describing the distribution of both low- $p_{T}$ and high- $p_{T}$ particles in high-multiplicity collisions [20-42]. Even models that assume strong-coupling dynamics for low- $p_{T}$ medium modes that are weakly coupled to high- $p_{T}$ modes have been argued as successfully describing LHC jet data [43-46].

\footnotetext{
*brnnad007@myuct.ac.za

†wa.horowitz@uct.ac.za
}

Published by the American Physical Society under the terms of the Creative Commons Attribution 4.0 International license. Further distribution of this work must maintain attribution to the author(s) and the published article's title, journal citation, and DOI. Funded by $S C O A P^{3}$.
In vacuum, quarkonia are bound states of a heavy quark and its antiquark pair, e.g., the $c \bar{c}$ pair in a $J / \psi$ meson or a $b \bar{b}$ pair in the $\Upsilon(1 \mathrm{~S})$ meson [47]. Embedded in a medium, the properties of quarkonia change. Matsui and Satz [48] were the first to propose that suppression of the $J / \psi$ meson spectrum should be observed in the QGP due to Debye screening of the color charge. Heavy quarkonia may theoretically exist in conjunction with the QGP at $T>T_{c}$, where $T_{c}$ is the critical temperature required for QGP formation, due to its small binding radii relative to the screening radius, whereas lighter hadrons dissociate at $\sim T_{c}$. At some $T$, the screening radius eventually becomes smaller than the typical heavy quarkonia radii, leading to their dissolution [49]. In addition, excited states of heavy quarkonia dissociate before the ground state [50]. The suppression of the bound states of heavy quarkonia in heavy-ion collisions is hence a valuable indicator of the formation of QGP, and the comparison of the quarkonia spectra in high-multiplicity collisions to that in minimum bias $p+p$ collisions where no QGP is formed is a useful probe of the QGP's properties.

Also pioneered in Ref. [48] was the use of potential models to describe the interaction of the quark and antiquark in the $q \bar{q}$ pair to calculate the suppression of quarkonia spectra in heavy-ion collisions. In these potential models, the large mass and small relative velocity of the heavy quarks justifies the use of nonrelativistic quantum mechanics (NRQM) to describe the quarkonia: The nonrelativistic Schrödinger equation gives a binding energy for the quarkonia given a model potential for the $q \bar{q}$ interaction.

Further works [51-55] have shown that in addition to a standard real Debye-screened term, the potential of heavy quarkonia at finite temperature contains an imaginary part which gives the thermal width of the state and hence its suppression. One of the first to do so was Ref. [51], which made use of perturbative methods to find the static potential of heavy quarkonia at finite temperature. They concluded that 
the thermal width of the state increases with temperature $T$, suggesting that at high $T$ the dissociation due to the effect described by the imaginary part of the potential occurs before color screening can even come into effect. Physical interpretations of this damping were provided by Refs. [52,53] in the form of inelastic scatterings of hard particles in the plasma with one another and surrounding gluons, with Ref. [56] suggesting that quarkonia suppression could also be attributed to $q \bar{q}$ color singlet to octet break-up even when the Debye mass is smaller than the quarkonia binding energy.

The complex-valued potential was explored further using nonperturbative lattice QCD by Refs. [54,55], allowing for the study of strongly coupled quarkonia as well. However, lattice techniques are restricted to quarkonia at or very near rest with respect to the medium and inverting lattice observables to the quarkonia potential is a nontrivial, possibly ill-defined process [57,58]; certainly another theoretical tool is required to investigate a quarkonium moving rapidly with respect to a strongly coupled medium.

The potential for static heavy quarkonia at finite temperature in $\mathcal{N}=4$ super Yang-Mills (SYM) theory was calculated via the methods of AdS/CFT in Refs. [59-64]. Liu, Rajagopal, and Wiedemann (LRW) [65] were the first to present a description from AdS/CFT of the consequences of velocity on the screening length of charmonium. They found that the plasma screening length decreased with velocity and therefore could result in a significant additional source of suppression at high transverse momentum $p_{T}$. Since then, Refs. [66-69] have performed similar investigations. While the aforementioned are limited in their scope of application, it is interesting to note that Ref. [66] in particular concludes that the effect of velocity may not be as consequential as postulated in LRW.

On the other hand, from pQCD, Ref. [70] found a potential for weakly coupled heavy quarkonium states which is dependent on velocity and shows that the dissociation energy increases with quarkonia velocity.

We would ultimately like to investigate the consequences of these different velocity-dependence pictures from AdS/CFT compared to pQCD. In this paper we have a more modest goal: to compare the experimentally measurable consequences of $\mathrm{pQCD}$ vs. AdS/CFT pictures for computing the quarkonia potential when the quarkonia are at rest with respect to the medium.

The usual observable used to compare theoretical predictions for quarkonia to data is the nuclear modification factor, $R_{A A}\left(\left\{x_{i}\right\}\right)$, which is the ratio of the number of quarkonia observed in an $A+A$ hadronic collision as a function of the set of variables $\left\{x_{i}\right\}$ to the number observed in minimum bias $p+p$ collisions, scaled by the number of $p+p$-like collisions in the $\left\{x_{i}\right\}$ collisions. Should the production processes for quarkonia remain unchanged in an $A+A$ collision, then an $R_{A A}$ less than 1 indicates a suppression of quarkonia and would indicate the presence of a medium that caused the quarkonia to dissociate.

One of the challenges of quarkonia research, however, is the significant number of unknowns that cloud the interpretation of $R_{A A}$. For example, even in $p+p$ collisions, the production mechanism for quarkonia is not under good theoretical control [57,58]; thus it is unclear how the production spectrum of quarkonia is affected quantitatively in $A+A$ collisions. By focusing on bottomonia, whose formation time $\sim 1 / 2 m_{b} \ll$ $\tau_{\text {form }}$, where $\tau_{\text {form }}$ is the formation time of the QGP medium in $A+A$ collisions, we hope to limit our theoretical uncertainty due to quarkonia formation physics.

Another complication is the possibility for regeneration. As $\sqrt{ } s$ and $T$ increase, the density of open charm in the medium increases. Then the possibility of quarkonia to spontaneously form from these charm combining or of dissociated quarkonia reforming through the capture of these open in-medium charm quarks increases. Since $m_{b} \gg T$ at RHIC and LHC and the hard production cross section for bottom is small enough, we can avoid considering regeneration in our bottomonia calculations [71].

In this paper we consider the case of ground-state bottomonia at rest with respect to an isotropic quark-gluon plasma. We follow the methodology outlined in Krouppa et al. [71], with a number of improvements which we denote NRQM. Given a potential, we evolve a random wave function through imaginary time; after a sufficiently long evolution, only the ground-state wave function remains. This ground-state wave function then determines the ground-state binding energy. We independently confirmed the results of the NRQM method both the binding energies from $\mathrm{pQCD}$ and AdS/CFT by an application of the complex Ritz variational method [72]. We further confirmed the strong-coupling binding energies by deriving the meson spectrum in AdS/CFT using semiclassical, rotating open strings by generalizing the methods of Kruczenski et al. [73] to $T>0$. Following Krouppa, we used the complex binding energies in a quarkonia suppression model to compute $R_{A A}$.

The potential models used are presented in Sec. II, taken from leading-order perturbative calculations in Ref. [71] for weakly coupled quarkonia and AdS/CFT calculations in Ref. [61] for strongly coupled quarkonia. These potentials are used to solve the nonrelativistic, time-dependent Schrödinger Equation (TDSE) for the ground-state energy and hence binding energy of $\Upsilon(1 S)$, using the imaginary time techniques as outlined in Sec. III. The resulting binding energies as a function of temperature are provided in Sec. IV and are independently confirmed using complex variational methods as laid out in Appendix A. An independent derivation of the meson spectrum in AdS/CFT using semiclassical, rotating open strings is presented in Appendix B. Our predictions for $R_{A A}$ as a function of $N_{\text {part }}$ and $p_{T}$, respectively, follows in Sec. V. We conclude our manuscript with a discussion and outlook in Sec. VI.

\section{POTENTIAL MODELS}

\section{A. Weakly coupled quarkonia}

The potential model presented here for weakly coupled quarkonia is taken from Ref. [71] and is complex valued as $V(r)=\operatorname{Re}[V(r)]+i \operatorname{Im}[V(r)]$. Both the real and the imaginary parts of the potential were found using leading-order perturbative calculations. The real part of the potential was derived from the Fourier transform of the gluon propagator in the real time formalism, and the imaginary part of the potential was derived from the symmetric propagator in the imaginary 
time formalism; these derivations are presented in Ref. [74] and Ref. [75], respectively.

The real part of the potential is given by

$$
\begin{aligned}
\operatorname{Re}[V(r)]= & -\frac{\alpha}{r}(1+\mu r) e^{-\mu r}+\frac{2 \sigma}{\mu}\left(1-e^{-\mu r}\right) \\
& -\sigma r e^{-\mu r}-\frac{0.8 \sigma}{m_{Q}^{2} r},
\end{aligned}
$$

where $r$ is the distance between the quark and antiquark in the $q \bar{q}$ pair, $\sigma=0.223 \mathrm{GeV}^{2}$, and $\alpha=0.385$. We take $m_{Q}=$ $4.7 \mathrm{GeV}$ for bottomonium. We take $\mu=m_{D}$, as was done in Ref. [71], where $m_{D}$ is the Debye mass,

$$
m_{D}^{2}=(1.4)^{2} N_{c}\left(1+N_{f} / 6\right) 4 \pi \alpha_{s} T^{2} / 3 .
$$

The factor of $(1.4)^{2}$ is an adjustment made to take into account higher-order corrections to the leading-order perturbative calculation of $m_{D}$ as determined using lattice calculations in [76].

We will take $m_{D} \simeq 3 p_{\text {hard }}=3 T$, as was done in Ref. [71], where $p_{\text {hard }}$ is a constant hard momentum scale for the particles in the QGP. With parameters $N_{c}=3, N_{f}=2$, and $\alpha_{s}=$ $3 \alpha / 4$ from the relation $\alpha \equiv C_{F} \alpha_{s}=\left[\left(N_{c}^{2}-1\right) /\left(2 N_{c}\right)\right] \alpha_{s}$, then Eq. (2) yields $m_{D}^{2}=9.48 T^{2} \simeq 9 p_{\text {hard }}^{2}$, as required.

We plot $\operatorname{Re}[V(r)]$ as a function of quark separation $r$ for various temperatures in Fig. 1(a). by

The imaginary part of the weakly coupled potential is given

$$
\operatorname{Im}[V(r)]=\alpha T \phi(\hat{r}),
$$

where $\hat{r} \equiv m_{D} r$ and

$$
\phi(\hat{r}) \equiv 2 \int_{0}^{\infty} d z \frac{z}{\left(z^{2}+1\right)^{2}}\left[1-\frac{\sin (z \hat{r})}{z \hat{r}}\right] .
$$

We plot $\operatorname{Im}[V(r)]$ as a function of quark separation $r$ for various temperatures in Fig. 1(b).

Note that, in order not to cut off the wave function prematurely, the potential must be considered at least as far as $20 \mathrm{fm}$. The function in Eq. (4) reduces in part to a Meijer G-function which becomes difficult to compute numerically for $r \gtrsim 10 \mathrm{fm}$. Hence, we imposed a maximum allowed value on $\hat{r}$ of $\hat{r}_{\max }=29.95$, which resulted in $\operatorname{Im}[V(r)]$ leveling off consistently as shown in Fig. 1(b). We found that our results are insensitive to the precise location of the maximum value of $\hat{r}_{\max }$ chosen.

\section{B. Strongly coupled quarkonia}

We modeled the strongly coupled heavy quarkonia at rest in a QGP with the potential given in Albacete et al. [61]. In that work, the authors derive the potential in $\mathcal{N}=4$ super Yang-Mills at finite temperature using AdS/CFT based on the methods of Rey et al. [59] and Brandhuber et al. [60].

The potential in Ref. [61] is given by

$V_{s}(r)=\frac{\sqrt{\lambda}}{2 c_{0} \pi}\left[-\frac{1}{z_{\max }}\left(1-\frac{z_{\max }^{4}}{z_{h}^{4}}\right) F\left(\frac{1}{2}, \frac{3}{4} ; \frac{1}{4} ; \frac{z_{\max }^{4}}{z_{h}^{4}}\right)+\frac{1}{z_{h}}\right]$,

where $\lambda$ is the 't Hooft coupling, $c_{0}=\Gamma^{2}\left(\frac{1}{4}\right) /(2 \pi)^{3 / 2}$, and $F$ is the usual Gaussian hypergeometric function. The temper-

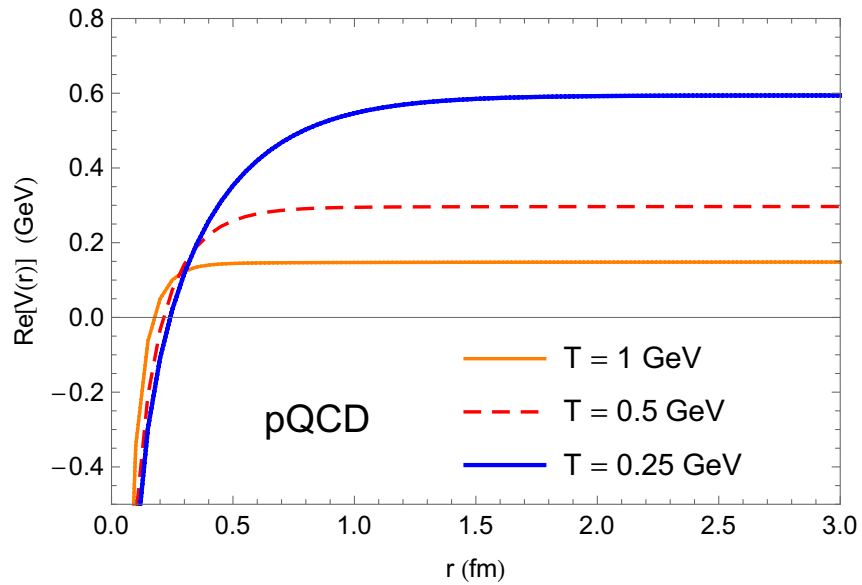

(a)

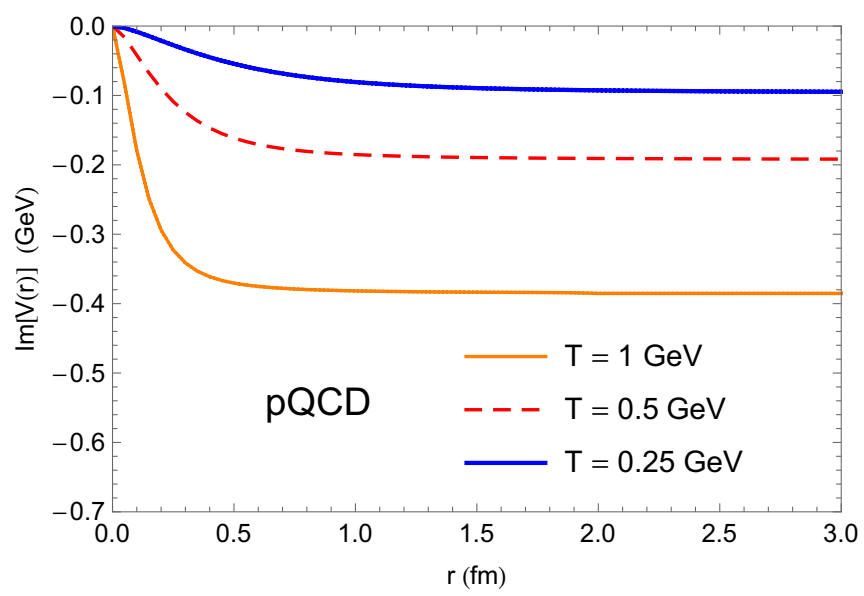

(b)

FIG. 1. Plot of the (a) real part of the potential $\operatorname{Re}[V(r)]$, given in Eq. (1), and the (b) imaginary part of the potential $\operatorname{Im}[V(r)]$, given in Eq. (3), as a function of the distance $r$ between the quark and antiquark in the $b \bar{b}$, for various $T$ in an isotropic plasma. $T=$ $0.25 \mathrm{GeV}$ is denoted by the thick blue line, $T=0.5 \mathrm{GeV}$ by the dashed red line and $T=1 \mathrm{GeV}$ by the thin orange line.

ature dependence comes from $z_{h}=1 / \pi T$, and $z_{\max }$ is found from the implicit equation

$$
r c_{0}=\frac{z_{\max }}{z_{h}^{2}} \sqrt{z_{h}^{4}-z_{\max }^{4}} F\left(\frac{1}{2}, \frac{3}{4} ; \frac{5}{4} ; \frac{z_{\max }^{4}}{z_{h}^{4}}\right) .
$$

Note that $z_{\max }$, and hence the potential, becomes complex for $r>r_{c} \simeq 0.870 z_{h}$. The original papers [59,60] abandon the solution at this point, but Ref. [61] does not. Considering that both weakly coupled pQCD and nonperturbative lattice QCD methods yield heavy quark potentials with both real and imaginary parts, it is sensible to expect the same using AdS/CFT methods. Further, allowing for complex $z_{\max }$ smooths out the kink in the potential from Refs. $[59,60]$ and does not violate any precepts of AdS/CFT.

We provide further supporting evidence for our procedure in Appendix B, which gives an independent calculation of the binding energies for $\Upsilon(1 \mathrm{~S})$ using semiclassical, rotating open strings attached to the D7-brane. For large angular 


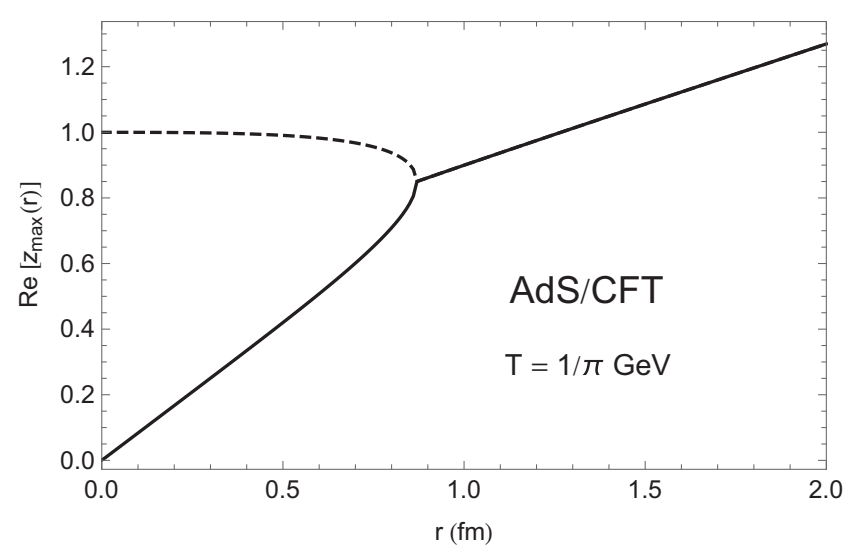

(a)

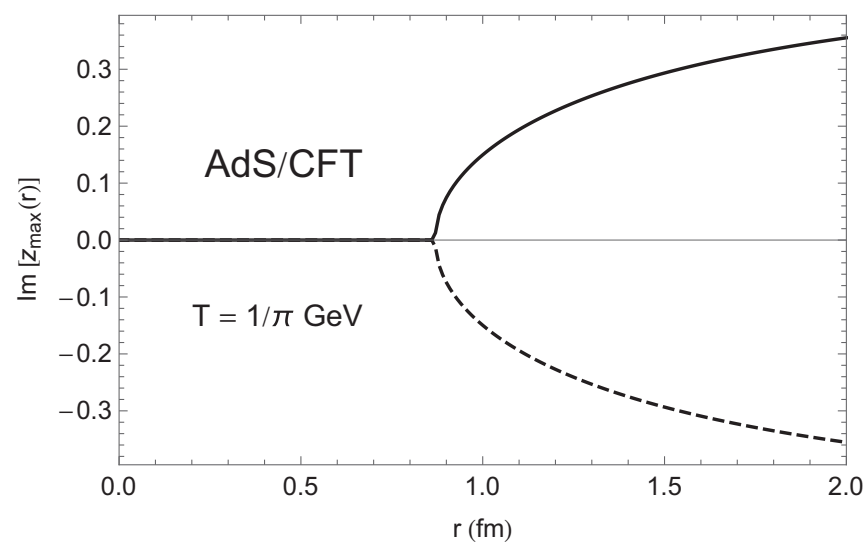

(b)

FIG. 2. All possible solutions for (a) $\operatorname{Re}\left[z_{\max }\right]$ and (b) $\operatorname{Im}\left[z_{\max }\right]$, as a function of quark separation $r$ for $T=1 / \pi \mathrm{GeV}$ from Eq. (6). The solid line is the root chosen over the dashed line solution for calculating the potential $V_{s}(r)$ as shown in Fig. 3.

momenta $J$, we expect these semiclassical methods to give the same (complex) binding energies as the NRQM method using the complex Albacete et al. [61] potential. We show in Appendix B that the two methods do converge for large $J$. We further show that at small $J$, the added physics of quantum mechanics in the NRQM method leads to a mild loosening of the meson compared to the semiclassical approach, as expected. ${ }^{1}$

Figure 2 shows the real and imaginary parts of $z_{\max }$, respectively, versus quark separation $r$ for $T=1 / \pi \mathrm{GeV}$.

The solution for $\operatorname{Re}\left[z_{\max }\right]$ denoted by the solid line is chosen as the physically relevant one, as was done in Ref. [61]. This choice is made because that solution agrees with the solution first found by Maldacena [77] for a heavy quark

\footnotetext{
${ }^{1}$ As was shown in Kruczenski et al. [73], formally for $\lambda \rightarrow \infty$ the $J=0$ binding energy for a quarkonium from AdS/CFT is on the order of the mass. Such a large binding energy implies relativistic motion of the $q \bar{q}$ pair, which invalidates the NRQM approach. However for the phenomenologically relevant values of $\sqrt{\lambda} \sim 3.5-5.5$, $E_{\text {bind }} \ll E_{m_{Q}}$. As is shown more fully in Appendix B, nonrelativistic methods are well suited even for $J=0$ for these values of $\lambda$.
}

potential in vacuum for $\mathcal{N}=4$ SYM theory, given in Eq. (7), which reduces to $z_{\max }=r c_{0}$ at zero temperature.

Furthermore, the solid root is chosen for $\operatorname{Im}\left[z_{\max }\right]$ over its complex conjugate. This choice is justified as follows. The time evolution of the wave function of any given state is $e^{-i E t} \sim e^{\operatorname{Im}[E] t}$. In order to ensure that the probability of a single state does not exceed one, we require that $\operatorname{Im}[E]<0$ [61], and therefore $\operatorname{Im}\left[V_{s}(r)\right]<0$. We thus choose the positive root for $\operatorname{Im}\left[z_{\max }\right]$, which yields the required negative $\operatorname{Im}\left[V_{s}(r)\right]$.

Figures 3(a) and 3(b) show the real and imaginary parts of $V_{s}(r)$ as a function of quark separation $r$, calculated using the roots chosen for $z_{\max }$ as shown in Fig. 2, and taking $\lambda=10$.

As mentioned earlier, Ref. [77] gives the heavy quark potential in a vacuum at zero temperature as

$$
V_{0}(r)=-\frac{\sqrt{\lambda}}{2 \pi c_{0}^{2} r} .
$$

The plot of $V_{0}(r)$ is given in Fig. 3(a) for comparison.

Note that the real parts of the pQCD and AdS/CFT potentials, shown in Figs. 1(a) and 3(a), respectively, are similar in form, but the imaginary parts from $\mathrm{pQCD}$ and AdS/CFT, shown in Figs. 1(b) and 3(b), respectively, differ greatly: The imaginary part of the pQCD potential saturates as a function of $r$, whereas that of the AdS/CFT potential diverges.

We also note that the potential above as derived from AdS/CFT is lacking an $r$-independent but $T$-dependent term that ensures that the potentials are identical in the $r T \rightarrow 0$ limit [78]. The consideration of such a $T$-dependent term results, however, in a potential identical to the one quoted above, barring some constant additive difference. Since the real part of the potential at infinity is ultimately subtracted from the binding energies, as discussed in Sec. III which follows, neglecting the $r$-independent but $T$-dependent term leaves the binding energies, and hence the suppression results, unchanged.

\section{NUMERICAL INTEGRATION OF TDSE}

The methodology used here follows that of Refs. $[71,79,80]$, adapted to the special case of an isotropic plasma, with various modifications of the discretization as explained further below. In order to compute the ground-state wave function, and hence the binding energy, we need to solve the nonrelativistic, TDSE in spherical coordinates, subject to a spherically symmetric wave function $\Psi=\Psi(r, t)$. The TDSE is thus

$$
i \partial_{t} \Psi(r, t)=H \Psi(r, t),
$$

where the Hamiltonian $H$ is

$$
\begin{aligned}
& H=-\frac{1}{2 m} \nabla^{2}+V(r), \\
& \nabla^{2}=\frac{1}{r^{2}} \frac{\partial}{\partial r}\left(r^{2} \frac{\partial}{\partial r}\right)+f(\theta, \phi),
\end{aligned}
$$

where $m \equiv m_{1} m_{2} /\left(m_{1}+m_{2}\right)$ is the reduced mass of the quarkonium. Note that $f(\theta, \phi)$ can be neglected since we are dealing with an isotropic quark gluon plasma. We take $\hbar=c=1$ throughout the paper. 


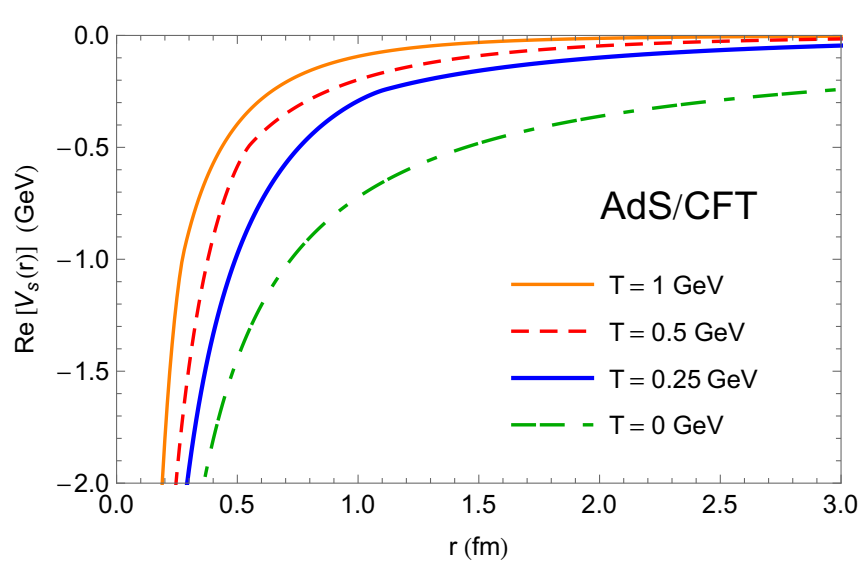

(a)

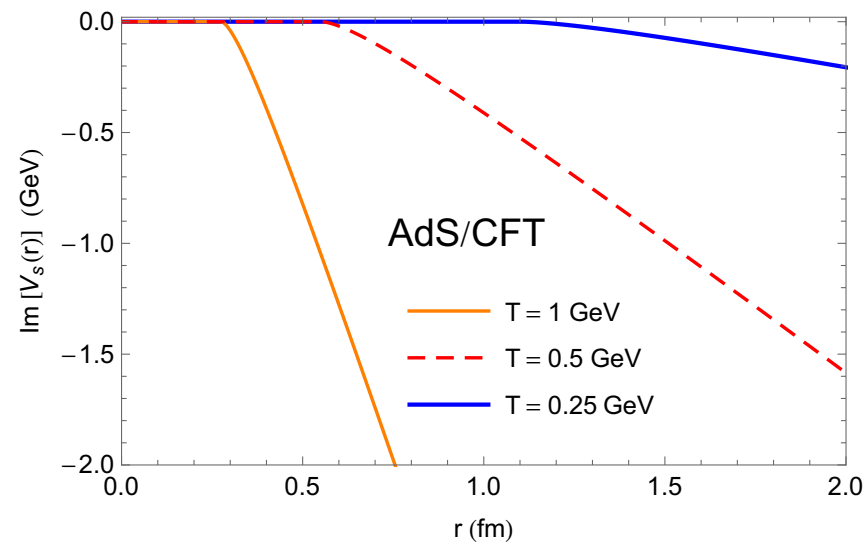

(b)

FIG. 3. The (a) real part of the strongly coupled potential $\operatorname{Re}\left[V_{s}(r)\right]$ and the (b) imaginary part of the potential $\operatorname{Im}\left[V_{s}(r)\right]$ from Eq. (5) as a function of the distance $r$ between the quark and antiquark in the $b \bar{b}$, for various temperatures $T$ in an isotropic plasma. $T=0.25 \mathrm{GeV}$ is the thick blue line, $T=0.5 \mathrm{GeV}$ the dashed red line and $T=1 \mathrm{GeV}$ the thin orange line. $T=0 \mathrm{GeV}$ is included in (a) as the dashed-dotted green line, taken from Eq. (7).

Performing a Wick rotation to an imaginary time $\tau \equiv i t$, Eq. (8) has the general solution

$$
\Psi(r, \tau)=\sum_{n=0}^{\infty} c_{n} \psi_{n}(r) e^{-E_{n} \tau} .
$$

Since $E_{n}>E_{0}$ for all $n>0$, one can evolve forward in imaginary time such that all the higher-order wave functions are suppressed and only the ground-state wave function remains:

$$
\lim _{\tau \rightarrow \infty} \Psi(r, t) \rightarrow c_{0} \psi_{0}(r) e^{-E_{0} \tau},
$$

where $\psi_{0}(r)$ is the ground-state wave function and $E_{0}$ the ground-state energy.

For simplicity, we will redefine the imaginary time to be dimensionless, $\tau \equiv \mathrm{itm}$, along with further dimensionless quantities $\rho, R(\rho)$, and $W(\rho)$,

$$
\rho \equiv m r, \quad R(\rho, \tau) \equiv r \Psi(r, \tau), \quad W(\rho) \equiv \frac{V(\rho)}{m} .
$$

The TDSE in terms of the dimensionless quantities is

$$
\frac{\partial}{\partial \tau} R(\rho)=\frac{1}{2}\left[\frac{\partial^{2}}{\partial \rho^{2}} R(\rho)\right]-W(\rho) R(\rho) .
$$

We improve on the finite-difference time domain scheme implemented in Ref. [79] (and elaborated on in Ref. [80]) by using a Crank-Nicolson Scheme, since this scheme is stable for much larger time steps $\Delta \tau$ [81]. Given a PDE of the form

$$
\frac{\partial u}{\partial t}=\frac{\partial}{\partial x}\left(D \frac{\partial u}{\partial x}\right)
$$

one can discretize it as follows:

$$
\begin{aligned}
\frac{u_{j}^{n+1}-u_{j}^{n}}{\Delta t}= & \frac{D}{2} \frac{\left(u_{j+1}^{n+1}-2 u_{j}^{n+1}+u_{j-1}^{n+1}\right)}{(\Delta x)^{2}} \\
& +\frac{D}{2} \frac{\left(u_{j+1}^{n}-2 u_{j}^{n}+u_{j-1}^{n}\right)}{(\Delta x)^{2}},
\end{aligned}
$$

where we use the notation

$$
u_{j+1}^{n+1} \equiv u[(n+1) \Delta t,(j+1) \Delta x] .
$$

Letting

$$
W(\rho) R(\rho)=W\left(\rho_{i}\right)\left(\frac{R_{i}^{n}+R_{i}^{n+2}}{2}\right),
$$

where we define $\tau_{n} \equiv(n-1) \Delta \tau$ and $\rho_{i} \equiv(i-1) \Delta \rho$, the discretization of Eq. (13) is then

$$
\begin{aligned}
& {\left[-\frac{\Delta \tau}{4(\Delta \rho)^{2}}\right] R_{i+1}^{n+1}+\left[1+\frac{2 \Delta \tau}{4(\Delta \rho)^{2}}+\frac{W\left(\rho_{i}\right) \Delta \tau}{2}\right] R_{i}^{n+1}} \\
& +\left[-\frac{\Delta \tau}{4(\Delta \rho)^{2}}\right] R_{i-1}^{n+1} \\
& =\left[\frac{\Delta \tau}{4(\Delta \rho)^{2}}\right] R_{i+1}^{n}+\left[1-\frac{2 \Delta \tau}{4(\Delta \rho)^{2}}-\frac{W\left(\rho_{i}\right) \Delta \tau}{2}\right] R_{i}^{n} \\
& +\left[\frac{\Delta \tau}{4(\Delta \rho)^{2}}\right] R_{i-1}^{n} \text {. }
\end{aligned}
$$

For the binding energy plots shown in Sec. IV, we used $\Delta r=0.01 \mathrm{fm}$ and $\Delta \tau=10(\Delta \rho)^{2}$, where we have that $\Delta \rho=$ $m \Delta r$. Note that for both the weakly and strongly coupled potentials, we implemented an $r_{\text {cut }}=10^{-5} / m_{Q}>0$ on the potentials to ensure that solutions did not blow up at $r=$ 0 . Details on extending the above methodology to consider $J>0$ are given in Appendix B 2 .

The binding energy of the state can then be found from

$$
E_{\text {bind }} \equiv E_{0}-\operatorname{Re}[V(|r| \rightarrow \infty)]
$$

where the ground-state energy $E_{0}$ can be found from the ground-state wave function,

$$
E_{0}=\frac{\int r^{2} d r \psi_{0}(r)^{*} H \psi_{0}(r)}{\int r^{2} d r\left|\psi_{0}\right|^{2}}
$$




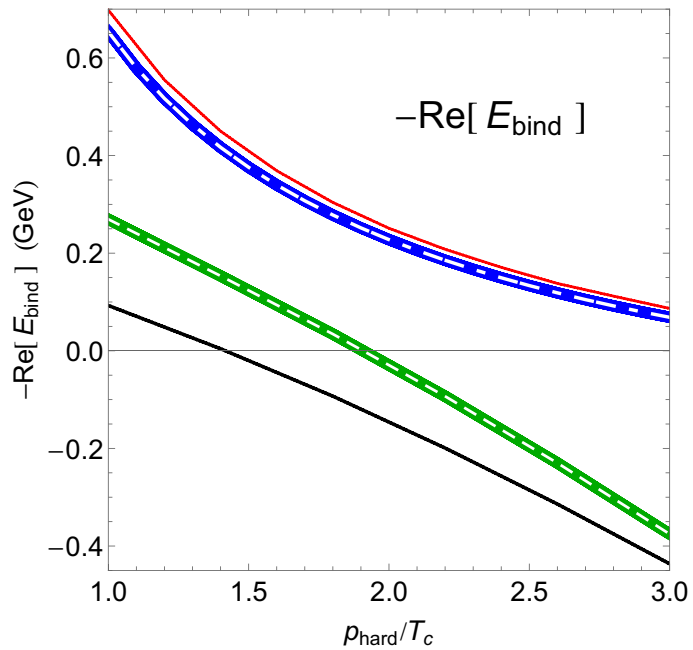

(a)

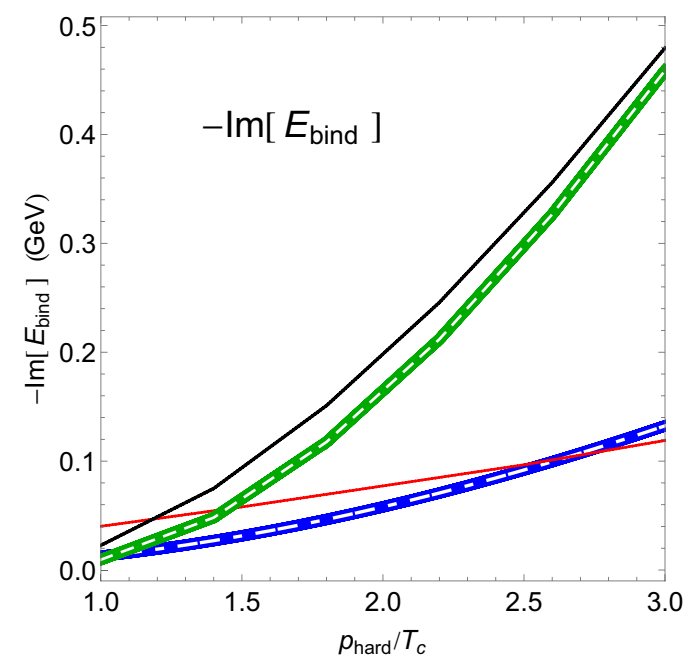

(b)

FIG. 4. The (a) negative real part of $E_{\text {bind }}$ and (b) negative imaginary part of $E_{\text {bind }}$ for $\Upsilon(1 S)$. The blue, green, and black curves give the results for weakly coupled and strongly coupled $(\lambda=10$ and $\lambda=5.5) \Upsilon(1 \mathrm{~S})$, respectively, computed from the imaginary time method of Sec. III. The dashed white curves inside the blue and green curves are from the independent evaluation using the complex variational method of Appendix A. The results from KRS [79], which should be identical to the blue curves, are given as solid red for comparison.

For weakly coupled quarkonia, the real part of the potential Eq. (1) at infinity reduces to a constant,

$$
\operatorname{Re}[V(|r| \rightarrow \infty)]=\frac{2 \sigma}{\mu} .
$$

In the case of the strongly coupled potential Eq. (5), $\operatorname{Re}\left[V_{s}(|r| \rightarrow \infty)\right]=0$.

\section{BINDING ENERGY RESULTS}

Figure 4(a) is a plot of the real part of the binding energy of $\Upsilon(1 S)$ from the pQCD potential, Eqs. (1) and (3), and strongly coupled potential, Eq. (5), as a function of temperature. Similarly, Fig. 4(b) gives the imaginary part of the binding energies for all cases mentioned above.

For the AdS/CFT results, we show the binding energy both for the case where the coupling constant is $\lambda=10$ (labeled as $\alpha_{s}=0.27$ ) and where $\lambda=5.5$; the reasoning behind the choice of these values is explained in Sec. V. The binding energy results for bottomonium from Ref. [79] are labeled "pQCD (KRS)" and are included for comparison.

Both the binding energy results presented for the pQCD potential and the AdS/CFT potential taking $\lambda=10$ were independently confirmed using a complex variational method, further explained in Appendix A.

The binding energy found from our adapted methodology for the pQCD potential, Eq. (1) and (3), differs quantitatively from that presented in Ref. [79], which was used in Krouppa et al. [71] to calculate suppression. In the case of $\Upsilon(1 \mathrm{~S})$, this difference does not change the qualitative behavior of the quarkonia, since both results suggest that the quarkonia remain bound up to at least $T=3 T_{c}$. However, we will see in Sec. V that the small quantitative differences in the derived binding energies lead to a significant quantitative difference in the predicted suppression.
In the case of ground-state charmonium, $J / \psi$, however, we see a qualitative difference in binding energies. The plot given in Ref. [79] agrees with the commonly accepted wisdom from lattice QCD that $J / \psi$ mesons cease to exist as a bound state between $1.5 T_{c}$ and $2.5 T_{c}$. However, using the same potential in Ref. [79] but with our methodology, we find that the $J / \psi$ do not dissociate in this temperature range.

That our results for the pQCD potential from Ref. [79] indicate bound charmonia above $T \gtrsim 3 T_{c}$ suggests a need either for an adjustment to the potential given in Ref. [71], since we believe our results to be more accurate than those presented in Ref. [79], or a slightly different approach in the interpretation of lattice QCD results with regards to the dissociation of $J / \psi$ at $T \sim 1.5 T_{c}$. We suspect that the plot presented in Ref. [79] resulted from the authors integrating over too small a spatial region (they indicated that they considered an $r_{\max }$ of $5 \mathrm{fm}$, whereas our results were sensitive to values of $r_{\max }$ as large as $\sim 15 \mathrm{fm}$ ).

The imaginary part of the binding energies from AdS/CFT are notably larger than those of weakly coupled quarkonia, and rise more steeply. This result is not surprising as the AdS/CFT potential has a divergent imaginary part, compared to the saturation of the imaginary part of the pQCD potential.

Unlike in the case of the weakly coupled quarkonia where the $\Upsilon(1 S)$ remains bound for the temperature range considered, the strongly coupled $\Upsilon(1 \mathrm{~S})$ dissociates at $\sim 1.9 T_{c}$. The comparatively larger imaginary part of the binding energy up to the temperature at which the bottomonium dissociates implies a much larger thermal width at higher $T$, and hence a larger suppression.

\section{SUPPRESSION}

We make quantitative predictions for the suppression of bottomonia in heavy-ion collisions and compare to 


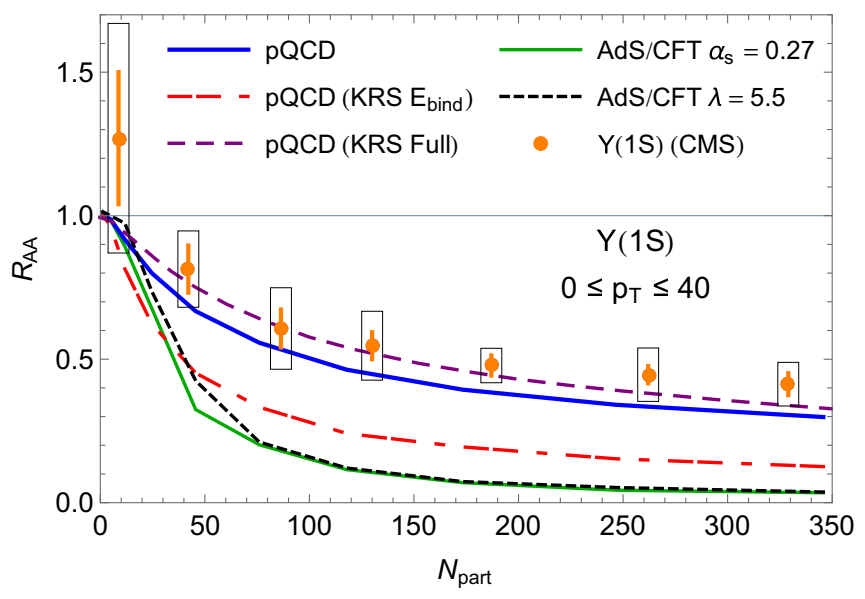

(a)

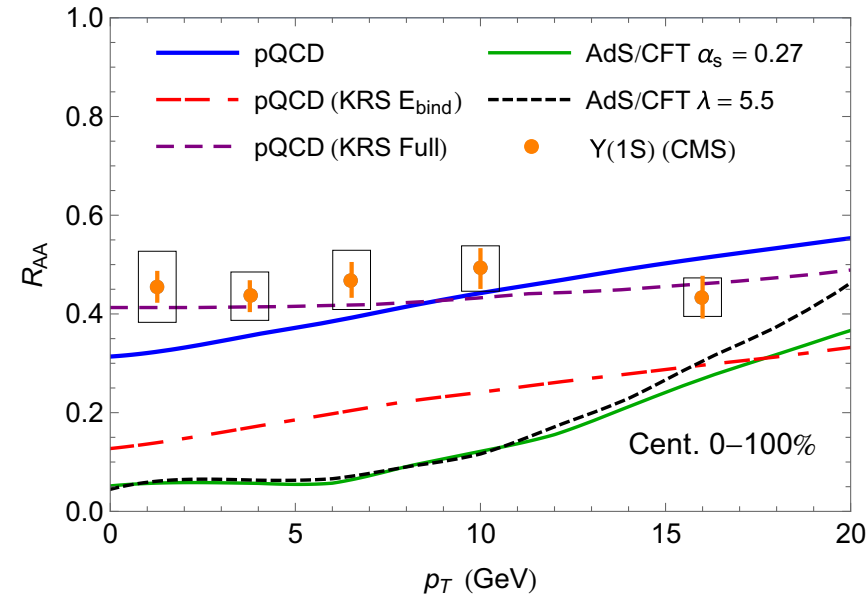

(b)

FIG. 5. (a) Nuclear modification factor $R_{A A}$ as a function of the number of participating nucleons $N_{\text {part }}$ for $0 \leqslant p_{T} \leqslant 40$. (b) Nuclear modification factor $R_{A A}$ as a function of transverse momentum $p_{T}$ for combined centrality classes. In both subfigures, the thick solid blue line gives our results for weakly coupled $\Upsilon(1 S)$, and the dashed-dotted red line that calculated for the binding energy from KRS [79] using our suppression model. The $R_{A A}$ presented in KRS [71] as calculated using their suppression model is given in dashed purple. The solid green and dotted black lines give the results for strongly coupled $\Upsilon(1 \mathrm{~S})$ with coupling constants $\alpha_{s}=0.27$ (and $T_{\mathrm{SYM}}=T_{\mathrm{QCD}}$ ) and $\lambda=5.5$ (and $\left.T_{\mathrm{SYM}}=T_{\mathrm{QCD}} / 3^{1 / 4}\right)$, respectively. Data from CMS [83] is included in orange.

measured data. The nuclear modification factor $R_{A A}$ is calculated following [71]:

$$
\begin{aligned}
& R_{A A}\left(p_{T}, y, \mathbf{x}_{\perp}, b\right)=e^{-\zeta\left(p_{T}, y, \mathbf{x}_{\perp}, b\right)}, \\
& \zeta \equiv \Theta\left(\tau_{f}-\tau_{\text {form }}\right) \int_{\max \left(\tau_{\text {form }}, \tau_{0}\right)}^{\tau_{f}} d \tau \Gamma\left(\tau, \mathbf{x}_{\perp}, \zeta=y\right),
\end{aligned}
$$

where the thermal width $\Gamma\left(\tau, \mathbf{x}_{\perp}, \varsigma\right)$ is given as

$$
\Gamma\left(\tau, \mathbf{x}_{\perp}, \varsigma\right)=\left\{\begin{array}{ll}
-2 \operatorname{Im}\left[E_{\text {bind }}\right] & \operatorname{Re}\left[E_{\text {bind }}\right]<0 \\
\gamma_{\text {dis }} & \operatorname{Re}\left[E_{\text {bind }}\right] \geqslant 0
\end{array} .\right.
$$

We take $\gamma_{\text {dis }}=10 \mathrm{GeV}$, as was done in Ref. [71]. Furthermore, $b$ is the impact parameter and $y$ the rapidity, taken to be zero. The formation time $\tau_{\text {form }}$ is calculated using

$$
\tau_{\text {form }}=E_{T} \tau_{\text {form }}^{0} / m_{Q},
$$

where $\tau_{\text {form }}^{0}=0.2 \mathrm{fm}$ is taken for the initial formation time of the state [71]. Last, the final time $\tau_{f}$ is taken as the time at which the temperature $T$ of the QGP drops below the critical temperature $T_{c}$.

We use the optical limit of the Glauber model [82] to describe the background in the case of $\sqrt{s_{N N}}=2.76-\mathrm{TeV}$ $\mathrm{Pb}+\mathrm{Pb}$ collisions. Taking a weighted average over the region with limits $\mathbf{x}_{\perp}=[-10,10] \mathrm{fm}$, we have

$$
R_{A A}\left(p_{T}, b\right)=\frac{\int d^{2} \mathbf{x}_{\perp} d \phi T_{A A}\left(\mathbf{x}_{\perp}, b\right) R_{A A}\left(p_{T}, y, \mathbf{x}_{\perp}, b\right)}{2 \pi N_{\text {coll }}},
$$

where $T_{A A}\left(\mathbf{x}_{\perp}, b\right)$ is the nuclear overlap function and $N_{\text {coll }} \equiv$ $\int d^{2} \mathbf{x}_{\perp} T_{A A}\left(\mathbf{x}_{\perp}, b\right)$ is the number of binary nucleon-nucleon collisions in the region.

We set a central temperature $T_{0}=522 \mathrm{MeV}$ and initial formation time $\tau_{0}=0.3 \mathrm{fm}$, as is done in Ref. [71]. Hence the temperature $T$ of the QGP is given by

$$
T\left(\tau_{0}, \mathbf{x}_{\perp}, b\right)=\beta\left[\frac{\rho_{\text {part }}\left(\tau_{0}, \mathbf{x}_{\perp}, b\right)}{\tau_{0}}\right]^{1 / 3},
$$

where $\beta=0.231 \mathrm{GeV}$ fm is a proportionality constant and $\rho_{\text {part }}$ the participant density.

Figure 5(a) gives the nuclear modification factor $R_{A A}$ for each of the sets of binding energies shown in Figs. 4(a) and 4(b) as a function of the number of participating nucleons $N_{\text {part }}$. To this end, the $R_{A A}\left(p_{T}, b\right)$ from Eq. (24) is averaged over the transverse momentum range $0 \leqslant p_{T} \leqslant 40$ with a weighting of $E^{-4}[71]$.

Figure 5(b) shows $R_{A A}\left(p_{T}\right)$, where all centrality classes are included, weighed by the number of binary nucleon-nucleon collisions $N_{\text {coll }}$.

Suppression results for midrapidity $(|y|<2.4) \mathrm{Pb}+\mathrm{Pb}$ collisions at $\sqrt{s_{N N}}=2.76 \mathrm{TeV}$ from the CMS Collaboration [83] are included in Figs. 5(a) and 5(b) for comparison.

We show in Figs. 5(a) and 5(b) two predictions for the suppression of strongly coupled bottomonia in an attempt to at least partially map out some of the systematic theoretical uncertainties associated with the use of the AdS/CFT correspondence. Since we used a potential derived in AdS-space dual to maximally supersymmetric Yang-Mills theory, there is no single obvious map between the parameters of QCD and of $\mathcal{N}=4 \mathrm{SYM}$. For the $\alpha_{s}=0.27$ curve, we took $\lambda_{\mathrm{SYM}}=10$ and $T_{\mathrm{SYM}}=T_{\mathrm{QCD}}$, where $\lambda_{\mathrm{SYM}}=10=\sqrt{4 \pi \alpha_{s} N_{c}}$ (and thus $\alpha_{s}=0.27$ for $N_{c}=3$ ) is approximately the value of the QCD running coupling constant evaluated at the first Matsubara frequency of the plasma. For the $\lambda=5.5$ curve, the coupling constant was set by a comparison to the $q \bar{q}$ potential from lattice and $T_{\mathrm{SYM}}=T_{\mathrm{QCD}} / 3^{-1 / 4}$ is a result of assuming the entropies of the QCD and SYM plasmas are the same [84]. 
We show in Figs. 5(a) and 5(b) three predictions for weakly coupled bottomonia: (1) the suppression using the binding energies we compute from the potential in Ref. [79] run through our medium background, (2) the suppression using the binding energies computed in Ref. [79] run through our medium background, and (3) the suppression quoted in Ref. [71] in which they run the binding energies computed in Ref. [79] through their background.

\section{DISCUSSION AND OUTLOOK}

In this paper we computed for the first time the suppression of bottomonia in an isotropic strongly coupled QGP and compared the results to those from a weakly coupled QGP and to data from the CMS Collaboration [83].

The nonrelativistic, time-dependent, radially symmetric Schrödinger Equation was solved numerically in order to find the ground-state wave functions for two potential models: one from $\mathrm{pQCD}$ and one from AdS/CFT. The discretized, numerical evaluation of the imaginary time Schrödinger equation was performed using a Crank-Nicolson Scheme, evolving forward in imaginary time until all higher-order wave functions were sufficiently suppressed. The potential used for weakly coupled quarkonia was taken from Ref. [79], in which the potential came from leading-order $\mathrm{pQCD}$ with various corrections. The strongly coupled quarkonia potential was taken from Ref. [61], who derived their potential from AdS/CFT.

The ground-state wave functions obtained were then used to find the (complex) ground-state binding energies for $\Upsilon(1 S)$. These ground-state energies were then independently confirmed using a complex variational technique [72]. Our binding energies for the weakly coupled potential in Ref. [79] differed somewhat from those found in Ref. [79], likely due to extending the physical region under consideration and from a possibly more careful treatment of the potential. For $\Upsilon(1 S)$, the difference was only quantitative, but for $J / \psi$ the difference was qualitative: For the potential in Ref. [79], we found charmonia remain bound up to at least $T \sim 3 T_{c}$.

Our first results for $\Upsilon(1 S)$ strongly coupled to a strongly coupled plasma show binding energies with much larger imaginary parts than those found from the pQCD potential, as well as real parts that become positive within the $T_{c}$ to $3 T_{c}$ range considered. Thus, for the potential models considered here, a strongly coupled $\Upsilon(1 \mathrm{~S})$ interacting with a strongly coupled plasma melts at a lower temperature than a weakly coupled $\Upsilon(1 S)$ interacting with a weakly coupled plasma. The $\Upsilon(1 \mathrm{~S})$ hence appears more strongly bound at weak coupling than at strong coupling, which is surprising. Since $\operatorname{Im}^{\mathrm{pQCD}}\left(E_{\text {bind }}\right)$ saturates for large $r$, cf. $\operatorname{Im}^{\mathrm{AdS}}\left(E_{\text {bind }}\right) \propto$ $r$, perhaps we are encountering a limitation of the pQCD calculation.

Since the weak-coupling bottomonia become more strongly bound as the coupling is increased and the strongcoupling bottomonia become less strongly bound as the coupling decreases, that the weak-coupling bottomonia is more strongly bound at weak coupling than at strong coupling suggests some nonmonotonic behavior of the binding energies at the threshold between the weak- and strong-coupling regimes. This nonmonotonic behavior possibly stems from deriving the potential at weak coupling in QCD, whereas the strongcoupling potential was derived in the slightly different theory, $\mathcal{N}=4$ SYM; it would be interesting to compare binding energies from the quarkonium potential at weak and strong coupling consistently within $\mathcal{N}=4$ SYM.

In an effort to support the validity of using complex heavy quark potentials from AdS/CFT in the calculation of the aforementioned binding energies, we also computed the meson spectrum of $\Upsilon(1 S)$ independently following the methodology outlined for $T=0$ by Kruczenski et al. [73], and extending the results to $T>0$. We considered semiclassical, rotating open strings attached to the D7-brane, which, in the $T=0$ large spin limit, describes two nonrelativistic quarks bound by a Coulomb potential.

We also generalized the strongly coupled potential from Ref. [61], which was derived in the infinite-mass limit, to finite mass. The binding energies from the semiclassical string method (SSM) and those from the imaginary time numerical method (NRQM) were the same in the semiclassical, large $J$ limit, as expected. At smaller $J$, the additional quantum effects incorporated in the NRQM method led to small differences in binding energies. These differences led to less tightly bound quarkonia, with less negative real parts of the binding energies from NRQM than the SSM, as well as greater imaginary parts of the binding energies from NRQM.

Even though the quarkonia in our imaginary time formalism is overall less tightly bound than that in the string picture from Ref. [73] due to consideration of quantum effects, the excellent agreement of the binding energies shows that the use of complex heavy quark potentials is consistent with an independent method of computing binding energies using AdS/CFT.

We input the complex ground-state binding energies we found into an implementation of the suppression model described in Ref. [71] to determine the $\Upsilon(1 \mathrm{~S})$ nuclear modification factor $R_{A A}$ as a function of the number of participating nucleons, $N_{\text {part }}$, and of transverse momentum, $p_{T}$, respectively. The difference in binding energies for the two coupling scenarios is echoed in the $R_{A A}$ results: From the larger imaginary parts of the strongly coupled binding energies, we see a significantly larger suppression for strongly coupled $\Upsilon(1 \mathrm{~S})$ than for weakly coupled $\Upsilon(1 \mathrm{~S})$. Quantitatively, our full model-composed of the potential, the resulting quarkonia binding energies, and the translation to $R_{A A}$ - significantly overpredicts the suppression of strongly coupled bottomonia compared to data. At the same time, our predictions for weakly coupled bottomonia are consistent with data.

We note that our model for the medium is significantly less sophisticated compared to that used in Ref. [71]: Our background is an optical Glauber model as opposed to the $3+1 \mathrm{D}$ viscous anisotropic hydrodynamics in that work. Our medium incorporates only Bjorken expansion, whereas the background in Ref. [71] includes transverse expansion and entropy production. Therefore the plasma in Ref. [71] cools faster than ours, leading to our model showing more dissociation for the same binding energies. The extent of the sensitivity of $R_{A A}$ to the background used is surprisingly large. With the only difference being the background geometry used, we ran the binding energies from Ref. [79] through our 
suppression model and found an $R_{A A}$ a factor of two smaller than that shown in Ref. [71].

In contrast to the favorable comparison between the pQCD-based results of Ref. [71] and the CMS data [7], if we assume our weak-coupling binding energies are more accurate than those of Ref. [79], then computing $R_{A A}$ with the more sophisticated background from Ref. [71] would likely yield a significant underprediction of the suppression of bottomonia.

At strong coupling, with a potential derived from AdS/CFT as described in Ref. [61], it seems unlikely that the use of a more sophisticated background would reduce the suppression of bottomonia enough that the predicted $R_{A A}$ would be consistent with data; however, the differences from using a more sophisticated background, suppression model, and velocity-dependent potential may ultimately be sufficient for future strongly coupled quarkonia predictions to be consistent with current data.

We note that our suppression calculations do not consider feed-down from higher excited states $\Upsilon(\mathrm{nS}), n>1$ for either the pQCD or AdS/CFT potentials. Considering feed-down, however, would only serve to suppress the $R_{A A}$ further. Hence, our qualitative conclusions about strongly coupled quarkonia would remain unchanged should higher excited states be included.

We leave the implementation of more advanced calculations of quarkonia suppression-including better modeling of the medium background, more accurate initial quarkonia production, a more realistic dissociation model, and the use of velocity-dependent potentials - and a more thorough investigation of systematic theoretical uncertainties in quarkonia $R_{A A}$ for future work.

\section{ACKNOWLEDGMENTS}

The authors thank the South African National Research Foundation and the SA-CERN Collaboration for their financial support. The authors also thank Michael Strickland for useful discussions.

\section{APPENDIX A: COMPLEX VARIATIONAL METHOD}

The complex binding energies presented in Sec. IV for the pQCD potential, as well as the AdS/CFT potential with a coupling constant of $\lambda=10$, were confirmed using the complex variational method presented here. We used the complex variational principle from Ref. [72], which can be seen for full theorems and proofs and is an extension of the usual Ritz variational method [85] to complex-valued potentials.

First, we define the c-product as

$$
(\psi \mid \phi)=\int_{\mathbb{R}^{n}} \psi(\vec{x}) \phi(\vec{x}) d^{n} x
$$

for two general functions $\psi(\vec{x})$ and $\phi(\vec{x})$. Then, given some eigenvalue problem $H \psi(\vec{x})=E \psi(\vec{x})$, where $(\psi \mid \psi) \neq 0$, we can define the Rayleigh quotient as

$$
R(\vec{\alpha}) \equiv \frac{(\psi|\hat{H}| \psi)}{(\psi \mid \psi)}
$$

where $\psi(\vec{x} ; \vec{\alpha})$ is a parameter-dependent trial wave function, with $\vec{\alpha} \in \mathbb{C}^{m}$.

In the event that $H \psi\left(\vec{x} ; \vec{\alpha}_{0}\right)=E \psi\left(\vec{x} ; \vec{\alpha}_{0}\right)$ is satisfied for some $\psi\left(\vec{x} ; \vec{\alpha}_{0}\right)$ and $\left(\psi\left(\vec{\alpha}_{0}\right) \mid \psi\left(\vec{\alpha}_{0}\right)\right) \neq 0$, then we have that

$$
\frac{\partial R\left(\vec{\alpha}_{0}\right)}{\partial \alpha_{0}^{i}}=0
$$

Note that the complex variational method only guarantees that $\psi\left(\vec{x} ; \vec{\alpha}_{0}\right)$ is a stationary wave function of the eigenvalue problem, not necessarily the ground state, as is the case with the real-valued potential Ritz variational method. Since the imaginary time techniques described in Sec. III guarantee a ground-state wave function if the imaginary time evolution is large enough, then a conclusion from these complex variational methods of the existence of a stationary state with binding energies equal to those from the imaginary time evolution is sufficient to confirm the imaginary time evolution ground-state binding energy results.

\section{APPENDIX B: COMPARISON TO AN INDEPENDENT ALTERNATIVE METHOD: HANGING, ROTATING STRINGS}

We seek an independent confirmation of the binding energies computed using the nonrelativistic imaginary time numerical method, given in Sec. III, using the potential from AdS/CFT as given in Sec. II B. We will do so by comparing the binding energies from our numerical method to those found using semiclassical, rotating open strings attached to the D7-brane.

Kruczenski et al. [73] computes the energy spectrum of mesons at $T=0$ from semiclassical, rotating open strings attached to a D7-brane. In particular, in the limit $J \gg \sqrt{\lambda}$, where $J$ is the spin, Ref. [73] notes that the spectrum corresponds to that of a nonrelativistic $q \bar{q}$ pair bound by a Coulomb potential. We extend the results from Ref. [73] to $T>0$ in Sec. B 1. In addition, we generalize the complex strongly coupled potential from Albacete et al. [61], which was derived in the infinite-mass limit, to finite mass in Sec. B 2. We show that the binding energies found using this generalized finite-mass potential with the nonrelativistic numerical regime from Sec. III, extended to $J>0$, is consistent with the binding energies from the semiclassical string method, in Sec. B 3.

\section{Semiclassical string solution}

In this section we follow the methodology of Kruczenski et al. [73] for $T=0$, in order to compute the meson energy spectrum as a function of $\operatorname{spin} J$ for $T>0$. Solving for the equation of motion of the string profile $\rho(z)$, we postulate a solution to the equations of motion (EOMs) of the form $\rho(z)=\rho_{\text {st. }}(z)+\delta \rho(z)$, where $\rho_{\text {st. }}(z)$ is the static string solution (as given in Sec. B 1 a) and $\delta \rho(z)$ a small first correction (found in Sec. B $1 \mathrm{~b}$ ). The energy and spin then follow in Sec. B 1 c.

We consider the simplest action describing the dynamics of an open string, i.e., the Nambu-Goto action,

$$
S_{\mathrm{NG}}=\frac{1}{2 \pi \alpha^{\prime}} \int d \tau d \sigma \sqrt{\left(\dot{X} \cdot X^{\prime}\right)^{2}-\dot{X}^{2} X^{\prime 2}},
$$


where $\tau$ and $\sigma$ are the coordinates parametrizing the string worldsheet, and $X^{\mu}=X^{\mu}(\tau, \sigma)$ specifies the embedding of the worldsheet in spacetime. We also have $\dot{X} \equiv \frac{d X}{d \tau}$ and $X^{\prime} \equiv$ $\frac{d X}{d \sigma}$, and the scalar products taken with respect to the $\operatorname{AdS}_{5} \times$ $\mathrm{S}_{5}$ metric [86], given as

$$
d s^{2}=\frac{R^{2}}{z^{2}}\left[-h(z) d t^{2}+d \rho^{2}+\rho^{2} d \theta^{2}+\frac{1}{h(z)} d z^{2}\right] .
$$

Here $\rho$ and $\theta$ are the usual spherical coordinates, and $z$ is the fifth string dimension, with the AdS boundary at $z=0$, the D7-brane situated at $z=z_{D 7}$, and the black hole horizon at $z=z_{h}$. We note that $R^{2}=\sqrt{\lambda} / \alpha^{\prime}$, where $R$ is the AdS radius and $\lambda$ the 't Hooft coupling. The dimensionless function $h(z) \equiv 1-z^{4} / z_{h}^{4}$ encodes the temperature dependence from the Hawking temperature $T=1 / \pi z_{h}$.

We are free to use the static gauge $\tau \equiv t$. In addition, we set $\theta=\omega t$ for constant $\omega$, constraining the string to rotate in the $\rho \theta$ plane. Hence, $X^{\mu}=(t, \rho(\sigma), \omega t, z(\sigma))^{\mu}$ and the action given in Eq. (B1) reduces to

$$
S=-\frac{R^{2}}{2 \pi \alpha^{\prime}} \int d t d \sigma \frac{1}{z^{2}} \sqrt{\left[h(z)-\omega^{2} \rho^{2}\right]\left[\rho^{\prime 2}+\frac{z^{\prime 2}}{h(z)}\right]} .
$$

The time-independent string profile is hence described by $\rho(\sigma)$ and $z(\sigma)$. Following Ref. [73], we define the dimensionless quantities $\tilde{\rho}(\sigma) \equiv \omega \rho(\sigma)$ and $\tilde{z}(\sigma) \equiv \omega z(\sigma)$ for convenience. The action can then be written as

$$
S=-\frac{R^{2} \omega}{2 \pi \alpha^{\prime}} \int d t d \sigma \frac{1}{\tilde{z}^{2}} \sqrt{\left[h(\tilde{z})-\tilde{\rho}^{2}\right]\left[\tilde{\rho}^{\prime 2}+\frac{\tilde{z}^{\prime 2}}{h(\tilde{z})}\right]}
$$

where $h(\tilde{z})=1-\tilde{z}^{4} / \tilde{z}_{h}^{4}$, with $\tilde{z}_{h} \equiv \omega z_{h}$. Using $S=\int d t L$, we can find the energy $E$ and the spin $J$, respectively:

$$
\begin{gathered}
E=\omega \frac{\partial L}{\partial \omega}-L \\
=\frac{R^{2} \omega}{2 \pi \alpha^{\prime}} \int \frac{d \sigma}{\tilde{z}^{2}} \sqrt{\frac{h(\tilde{z})\left(\tilde{z}^{\prime 2}+h(\tilde{z}) \tilde{\rho}^{\prime 2}\right)}{h(\tilde{z})-\tilde{\rho}^{\prime 2}}} \\
J=\frac{\partial L}{\partial \omega}=\frac{R^{2}}{2 \pi \alpha^{\prime}} \int d \sigma \frac{\tilde{\rho}^{2}}{\tilde{z}^{2}} \sqrt{\frac{\tilde{z}^{\prime 2}+h(\tilde{z}) \tilde{\rho}^{\prime 2}}{h(\tilde{z})\left(h(\tilde{z})-\tilde{\rho}^{\prime 2}\right)}}
\end{gathered}
$$

In order to solve for the string profile, we derive the EOMs. We choose $\sigma \equiv \tilde{z}$ in order to solve for $\tilde{\rho}(\tilde{z})$, as illustrated in Fig. 6.

Varying the action yields the Euler-Lagrange equation:

$$
\frac{\partial \mathcal{L}}{\partial \tilde{\rho}}-\frac{d}{d t} \frac{\partial \mathcal{L}}{\partial \dot{\tilde{\rho}}}-\frac{d}{d \tilde{z}} \frac{\partial \mathcal{L}}{\partial \tilde{\rho}^{\prime}}=0,
$$

where

$$
\mathcal{L}=-\frac{R^{2} \omega}{2 \pi \alpha^{\prime} \tilde{z}^{2}} \sqrt{\left[h(\tilde{z})-\tilde{\rho}^{2}\right]\left[\tilde{\rho}^{\prime 2}+\frac{\tilde{z}^{\prime 2}}{h(\tilde{z})}\right]}
$$

The second term of Eq. (B7) evaluates to zero, since $\mathcal{L}$ is independent of $\dot{\tilde{\rho}}$. We also note that since we are taking $J \gg$ $\sqrt{\lambda}$, we are in the limit $\omega \rightarrow 0$ [73]. Solving for the EOM to

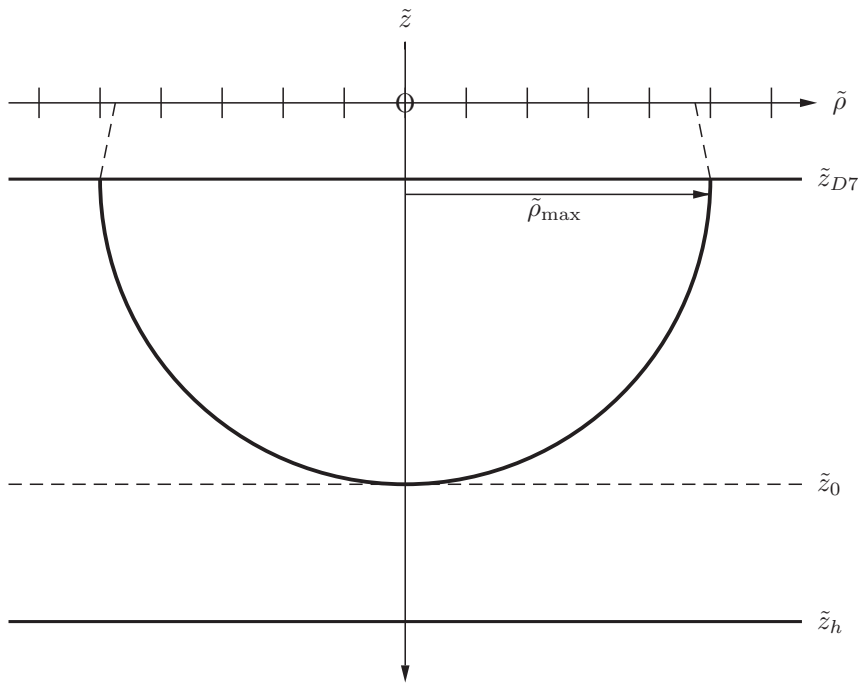

FIG. 6. The string profile $\tilde{\rho}(\tilde{z})$ in the limit $\omega \rightarrow 0$. The boundary of AdS space is situated at $\tilde{z}=0$, the D7-brane at $\tilde{z}=\tilde{z}_{D 7}$, and the black hole horizon at $\tilde{z}=\tilde{z}_{h}$. The turning point of the string is given by $\tilde{z}_{0}$, with $\tilde{\rho}_{\max }$ the radial distance at which the string is attached to the D7-brane. Shown also is the continuation of the solution beyond the D7-brane to the AdS boundary, i.e., $\tilde{\rho}\left(\tilde{z}_{D 7}\right) \simeq \tilde{\rho}(0)$.

next to leading order in $\omega$ then yields

$$
\begin{gathered}
\frac{\tilde{\rho}^{\prime \prime}}{1+h(\tilde{z}) \tilde{\rho}^{\prime 2}}-\frac{2}{\tilde{z}} \tilde{\rho}^{\prime}+\frac{\tilde{\rho}}{h(\tilde{z})\left(h(\tilde{z})-\tilde{\rho}^{2}\right)} \\
+\frac{h^{\prime}(\tilde{z}) \tilde{\rho}^{\prime}\left(2+h(\tilde{z}) \tilde{\rho}^{\prime 2}\right)}{2 h(\tilde{z})\left(1+h(\tilde{z}) \tilde{\rho}^{\prime 2}\right)}=0 .
\end{gathered}
$$

We will exploit that as $\omega \rightarrow 0$, one can expect that the string rotates very slowly, and the solution $\tilde{\rho}(\tilde{z})$ of Eq. (B9) is well approximated by that of a static string, $\tilde{\rho}_{\text {st. }}(\tilde{z})$. We will therefore set $\tilde{\rho}(\tilde{z})=\tilde{\rho}_{\text {st. }}(\tilde{z})+\widetilde{\delta \rho}(\tilde{z})$, where $\delta \rho \sim \omega^{3}$ is a small first correction. We solve for the static string solution $\tilde{\rho}_{\text {st. }}(\tilde{z})$ in Sec. B 1 a. The first correction $\widetilde{\delta \rho}(\tilde{z})$ is then found in Sec. B $1 \mathrm{~b}$ by systematically expanding in orders of the dimensionless parameter $\tilde{z}_{0}$.

\section{a. Static string solution $(\omega=0)$}

Let us solve first for the static string solution $\rho_{\text {st. }}(z)$. Since $\omega=0$, we will work in the untilded coordinates and then show that the solution $\rho_{\text {st. }}(z)$ can be written in terms of tilded coordinates while still valid for $\omega=0$.

Consider the action from Eq. (B3) for the case $\omega=0$, i.e., $\rho(z)=\rho_{\text {st. }}(z)$ :

$$
S=-\frac{R^{2}}{2 \pi \alpha^{\prime}} \int d t d \sigma \frac{1}{z^{2}} \sqrt{z^{\prime 2}+h(z) \rho_{\mathrm{st}}^{\prime 2}} .
$$

Setting $\sigma \equiv z$, we have the Lagrangian

$$
\mathcal{L}=-\frac{R^{2}}{2 \pi \alpha^{\prime} z^{2}} \sqrt{1+h(z) \rho_{\text {st. }}^{\prime 2}(z)}
$$

Note that $\mathcal{L}$ is independent of $\rho_{\text {st. }}(z)$. We can therefore, instead of solving for the equations of motion conventionally, define 
a conserved momentum

$$
\beta \equiv \frac{\partial \mathcal{L}}{\partial \rho_{\text {st. }}^{\prime}},
$$

which allows us to solve for $\rho_{\text {st. }}(z)$ using the boundary conditions of the string. Our conserved momentum, found substituting Eq. (B11) into Eq. (B12), is

$$
\beta=-\frac{R^{2}}{2 \pi \alpha^{\prime} z^{2}} \frac{h(z) \rho_{\mathrm{st} .}^{\prime 2}(z)}{\sqrt{1+h(z) \rho_{\mathrm{st} .}^{\prime 2}(z)}} .
$$

Solving Eq. (B13) for $\rho_{\text {st. }}^{\prime}(z)$, we have

$$
\rho_{\text {st. }}^{\prime}(z)= \pm \frac{2 \pi \alpha^{\prime} \beta z^{2}}{\sqrt{h(z)\left[R^{4} h(z)-\left(2 \pi \alpha^{\prime}\right)^{2} \beta^{2} z^{4}\right]}} .
$$

We note that the string has its turning point at $z=z_{0}$, i.e., $\rho_{\text {st. }}^{\prime}\left(z_{0}\right)=\infty$; hence the denominator of Eq. (B14) must vanish for $z=z_{0}$. This yields a solution for our conserved momentum:

$$
\beta= \pm \frac{R^{2}}{2 \pi \alpha^{\prime}} \frac{\sqrt{z_{h}^{4}-z_{0}^{4}}}{z_{h}^{2} z_{0}^{2}} .
$$

Choosing $\beta>0$ and $\tilde{\rho}_{\text {st. }}^{\prime}(z)<0$, i.e., $0 \leqslant \tilde{\rho}_{\text {st. }} \leqslant \rho_{\max }$, Eq. (B14) becomes

$$
\rho_{\text {st. }}^{\prime}(z)=-\frac{z^{2} \sqrt{z_{h}^{4}-z_{0}^{4}}}{\sqrt{\left(z_{h}^{4}-z^{4}\right)\left(z_{0}^{4}-z^{4}\right)}} .
$$

Noting that $\int_{z 0}^{z} d x \rho_{\text {st. }}^{\prime}(x)=\rho_{\text {st. }}(z)-\rho_{\text {st. }}\left(z_{0}\right)$, we have the static string solution

$$
\rho_{\text {st. }}(z)=\int_{z}^{z_{0}} d x \frac{x^{2} \sqrt{z_{h}^{4}-z_{0}^{4}}}{\sqrt{\left(z_{0}^{4}-x^{4}\right)\left(z_{h}^{4}-x^{4}\right)}} .
$$

We require the static string solution $\tilde{\rho}_{\text {st. }}(\tilde{z})$, however, both for the full solution $\tilde{\rho}(\tilde{z})=\tilde{\rho}_{\text {st. }}(\tilde{z})+\widetilde{\delta \rho}(\tilde{z})$ and in order to solve for the first correction $\tilde{\delta \rho}(\tilde{z})$ in Sec. B 1 b below. In terms of our dimensionless quantities $\tilde{\rho}$ and $\tilde{z}$, then Eq. (B17) can be written as

$$
\tilde{\rho}_{\text {st. }}(\tilde{z})=\int_{\tilde{z}}^{\tilde{z}_{0}} d x \frac{x^{2} \sqrt{\tilde{z}_{h}^{4}-\tilde{z}_{0}^{4}}}{\sqrt{\left(\tilde{z}_{0}^{4}-x^{4}\right)\left(\tilde{z}_{h}^{4}-x^{4}\right)}} .
$$

The $\omega$ 's implicit in Eq. (B18) cancel, hence leaving $\tilde{\rho}_{\text {st. }}(\tilde{z})$ valid in the limit $\omega \rightarrow 0$ in which it was derived.

\section{b. Correction $\widetilde{\delta \rho}(\tilde{z})$ to static string solution}

We now solve for the first correction $\widetilde{\delta \rho}(\tilde{z})$ by substituting the ansatz $\tilde{\rho}(\tilde{z})=\tilde{\rho}_{\text {st. }}(\tilde{z})+\widetilde{\delta \rho}(\tilde{z})$ into Eq. (B9), and neglecting terms $\mathcal{O}\left(\tilde{\delta \rho}^{2}\right)$. We note that $0<\tilde{z}<\tilde{z}_{0} \ll 1$ [73], and hence we can safely choose to expand Eq. (B9) in the small dimensionless parameter $\tilde{z}_{0}$.

In order to avoid expanding in quantities that are not necessarily small, we will define and work in the dimensionless parameters $\xi \equiv \tilde{z} / \tilde{z}_{0}$ and $\delta \equiv \tilde{z}_{0} / \tilde{z}_{h}$, which are not necessarily much smaller than 1 . We also define $\gamma \equiv \tilde{z}_{D 7} / \tilde{z}_{0} \ll 1$, where we have assumed that $\tilde{z}_{D 7} \ll \tilde{z}_{0}$-an assumption that is shown to be self-consistent later.

Making the change of coordinates $y \equiv x / \tilde{z}_{0}$, the static solution given by Eq. (B18) can be written as

$$
\tilde{\rho}_{\text {st. }}(\xi)=\tilde{z}_{0} \int_{\xi}^{1} d y \frac{y^{2} \sqrt{1-\delta^{4}}}{\sqrt{\left(1-y^{4}\right)\left(1-\delta^{4} y^{4}\right)}} .
$$

Clearly, $\tilde{\rho}_{\text {st. }} \sim \tilde{z}_{0}$. We note that $\widetilde{\delta \rho} \sim \tilde{z}_{0}^{2}$ admits only the trivial solution for the EOM; hence we take $\tilde{\delta \rho} \sim \tilde{z}_{0}^{3}$. Equation (B9) to leading order in $\tilde{z}_{0}$ is then:

$$
\begin{aligned}
& \widetilde{\delta \rho^{\prime \prime}}(\xi)+\widetilde{\delta \rho^{\prime}}(\xi)\left[\frac{2\left(\xi^{4} \delta^{4}\left(\xi^{4}+2\right)-2 \xi^{4}-1\right)}{\xi\left(1-\xi^{4}\right)\left(1-\xi^{4} \delta^{4}\right)}\right] \\
& =-\frac{\tilde{\rho}_{\text {st. }}(\xi)}{h(\xi)^{2}}\left[\tilde{z}_{0}^{2}+h(\xi) \tilde{\rho}_{\text {st. }}^{\prime 2}(\xi)\right],
\end{aligned}
$$

where we used that the static solution satisfies Eq. (B9) to leading order. Note that primed derivatives denote a derivative with respect to $\xi$.

The differential equation Eq. (B20) takes the general form

$$
\widetilde{\delta \rho^{\prime \prime}}(\xi)+f(\xi) \widetilde{\delta \rho^{\prime}}(\xi)=g(\xi),
$$

which can be rewritten as

$$
\begin{aligned}
& \frac{d}{d \xi}\left[\widetilde{\delta \rho^{\prime}}(\xi) e^{\int^{\xi} d x f(x)}\right]=g(\xi) e^{\int^{\xi} d x f(x)} \\
& \Rightarrow \quad \widetilde{\delta \rho^{\prime}}(\xi) e^{\int^{\xi} d x f(x)}=\int^{\xi} d x g(x) e^{\int^{x} d y f(y)} .
\end{aligned}
$$

Defining for convenience

$$
Q^{\prime}(\xi) \equiv \exp \left[-\int^{\xi} d x f(x)\right]
$$

we can write Eq. (B22) as

$$
\widetilde{\delta \rho^{\prime}}(\xi)=\frac{d}{d \xi}[\widetilde{\delta \rho}(\xi)]=Q^{\prime}(\xi) \int^{\xi} d x \frac{g(x)}{Q^{\prime}(x)} .
$$

It follows that

$$
\widetilde{\delta \rho}(\xi)=\int^{\xi} d x Q^{\prime}(x) \int^{x} d y \frac{g(y)}{Q^{\prime}(y)} .
$$

We note that by the chain rule

$$
\begin{aligned}
\frac{d}{d x}[Q(x)] \int^{x} d y \frac{g(y)}{Q^{\prime}(y)}= & \frac{d}{d x}\left[Q(x) \int^{x} d y \frac{g(y)}{Q^{\prime}(y)}\right] \\
& -Q(x) \frac{d}{d x}\left[\int^{x} d y \frac{g(y)}{Q^{\prime}(y)}\right] .
\end{aligned}
$$

Substituting Eq. (B26) into Eq. (B25) and simplifying, we can write Eq. (B25) as

$$
\widetilde{\delta \rho}(\xi)=Q(\xi) \int^{\xi} d x \frac{g(x)}{Q^{\prime}(x)}-\int^{\xi} d x Q(x) \frac{g(x)}{Q^{\prime}(x)} .
$$

Simplifying, the general solution is then

$$
\widetilde{\delta \rho}(\xi)=\int^{\xi} d x \frac{Q(\xi)-Q(x)}{Q^{\prime}(x)} g(x) .
$$


Using the explicit forms of $f(\xi)$ and $g(\xi)$ found by comparing Eq. (B20) and Eq. (B21), we can write the solution using the boundary conditions $\widetilde{\delta \rho}(1)=\widetilde{\delta \rho^{\prime}}(1)=0$ as:

$$
\widetilde{\delta \rho}(\xi)=-\int_{1}^{\xi} d x \frac{Q(\xi)-Q(x)}{Q^{\prime}(x)} \tilde{\rho}_{\text {st. }}(x) \frac{\tilde{z}_{0}^{2}+h(x) \tilde{\rho}_{\text {st. }}^{\prime 2}(x)}{h(x)^{2}},
$$

where

$$
Q(\xi)=\frac{\left(1-\gamma^{4}\right)^{3 / 2}}{\gamma^{2} \sqrt{1-\gamma^{4} \delta^{4}}} \int^{\xi} d x \frac{x^{2} \sqrt{1-x^{4} \delta^{4}}}{\left(1-x^{4}\right)^{3 / 2}} .
$$

We now wish to relate the D7-brane coordinate $\tilde{z}_{D 7}$, implicit in $\gamma=\tilde{z}_{D 7} / \tilde{z}_{0}$, to the coordinates $\tilde{z}_{0}$, and $\tilde{z}_{h}$, implicit in $\xi$ and $\delta$. To this end, we require that the string end orthogonally on the D7-brane:

$$
\tilde{\rho}^{\prime}(\gamma)=\tilde{\rho}_{\text {st. }}^{\prime}(\gamma)+\widetilde{\delta \rho}(\gamma)=0 .
$$

Taking the derivatives of $\tilde{\rho}_{\text {st. }}(\xi)$ and $\widetilde{\delta \rho}(\xi)$, as given in Eq. (B19) and (B29), we substitute into Eq. (B31) and expand the expression in $\gamma \ll 1$ where possible. Doing so leads to

$$
\tilde{z}_{0} \int_{\gamma}^{1} d y \frac{\sqrt{1-y^{4}}}{y^{2}\left(1-y^{4} \delta^{4}\right)^{3 / 2}} \tilde{\rho}_{\text {st. }}(y)=\sqrt{1-\delta^{4}} .
$$

To simplify further, we note that in the case $\omega \rightarrow 0$, we can argue that the full string solution $\tilde{\rho}(\xi)$ on the D7-brane is well approximated by the static string solution on the AdS boundary, i.e., $\tilde{\rho}(\gamma) \simeq \tilde{\rho}_{\text {st. }}(0)$. Hence we will set $\tilde{\rho}_{\text {st. }}(y) \simeq$ $\tilde{\rho}_{\text {st. }}(0)$ in Eq. (B32). From Eq. (B19) we can calculate $\tilde{\rho}_{\text {st. }}(0)$ explicitly:

$$
\tilde{\rho}_{\text {st. }}(0)=C_{0}(\delta) \tilde{z}_{0} \sqrt{1-\delta^{4}},
$$

where we have defined for convenience

$$
C_{0}(\delta) \equiv C_{2} F_{1}\left(\frac{1}{2}, \frac{3}{4}, \frac{5}{4}, \delta^{4}\right), \quad C \equiv \frac{\sqrt{2} \pi^{3 / 2}}{\Gamma(1 / 4)^{2}} .
$$

Note that ${ }_{2} F_{1}$ is the usual hypergeometric function. Setting $\tilde{\rho}_{\text {st. }}(y) \simeq \tilde{\rho}_{\text {st. }}(0)$ in Eq. (B32), we integrate to find

$$
\frac{\tilde{z}_{0} \tilde{\rho}_{\text {st. }}(0)}{\gamma}=\sqrt{1-\delta^{4}}+\mathcal{O}(\gamma) \Longrightarrow \frac{C_{0}(\delta) \tilde{z}_{0}^{2}}{\gamma} \simeq 1 .
$$

Substituting $\tilde{z}_{D 7}=\gamma \tilde{z}_{0}$, then, we have

$$
\omega \simeq \frac{C_{0}(\delta) \tilde{z}_{0}^{3}}{z_{D 7}} .
$$

We note that $\tilde{z}_{D 7} \sim \tilde{z}_{0}^{3}$, which leaves the $\tilde{z}_{D 7} \ll \tilde{z}_{0}$ assumption self-consistent. Writing Eq. (B30) in terms of $\tilde{z}_{0}, \delta$, and $\xi$ only, and expanding in $\tilde{z}_{0}$ as before, we have

$$
\begin{aligned}
Q(\xi) & =\frac{\left(1-C_{0}(\delta)^{4} \tilde{z}_{0}^{8}\right)^{3 / 2}}{C_{0}(\delta)^{2} \tilde{z}_{0}^{3} \sqrt{1-C_{0}(\delta)^{4} \delta^{4} \tilde{z}_{0}^{8}}} \int^{\xi} d x \frac{x^{2} \sqrt{1-\delta^{4} x^{4}}}{\left(1-x^{4}\right)^{3 / 2}} \\
& =\frac{1}{C_{0}(\delta)^{2} \tilde{z}_{0}^{3}} \int^{\xi} d x \frac{x^{2} \sqrt{1-\delta^{4} x^{4}}}{\left(1-x^{4}\right)^{3 / 2}}+\mathcal{O}\left(\tilde{z}_{0}^{-2}\right) .
\end{aligned}
$$

\section{c. Energy and spin}

Writing the energy $E$ from Eq. (B5) in terms of the dimensionless quantities $\xi$ and $\delta$, we can substitute $\tilde{\rho}(\xi)=$ $\tilde{\rho}_{\text {st. }}(\xi)+\widetilde{\delta \rho}(\xi)$ from Eqs. (B19) and (B29) to find:

$$
\begin{aligned}
E= & \frac{R^{2} \omega}{\pi \alpha^{\prime} \tilde{z}_{0}}\left\{\int_{C_{0}(\delta) \tilde{z}_{0}^{2}}^{1} \frac{d \xi}{\xi^{2}}\left[1+\frac{\tilde{\rho}_{\text {st. }}^{2}(0)}{2 h(\xi)}\right] \sqrt{1+h(\xi) \tilde{\rho}_{\text {st. }}^{\prime 2}(\xi)}\right. \\
& \left.+\int_{C_{0}(\delta) \tilde{z}_{0}^{2}}^{1} \frac{d \xi}{\xi^{2}} \frac{\tilde{\rho}_{\text {st. }}^{\prime}(\xi) \widetilde{\delta \rho^{\prime}}(\xi)}{\sqrt{1+h(\xi) \tilde{\rho}_{\text {st. }}^{\prime 2}(\xi)}}\right\} .
\end{aligned}
$$

To next to leading order in $\tilde{z}_{0}$, then, the energy is

$$
\begin{aligned}
E \simeq & \frac{R^{2}}{\pi \alpha^{\prime} z_{\mathrm{D} 7}}\left[1+\frac{C^{2} \tilde{z}_{0}^{2}}{2}\left(1-\delta^{4}\right){ }_{2} F_{1}\left(\frac{1}{2}, \frac{3}{4}, \frac{5}{4}, \delta^{4}\right)\right. \\
& \left.\times\left\{3{ }_{2} F_{1}\left(\frac{1}{2}, \frac{3}{4}, \frac{5}{4}, \delta^{4}\right)-4{ }_{2} F_{1}\left(\frac{3}{4}, \frac{3}{2}, \frac{5}{4}, \delta^{4}\right)\right\}\right] .
\end{aligned}
$$

Similarly the spin $J$ can be found using Eq. (B6):

$$
\begin{aligned}
J= & \frac{R^{2} \tilde{\rho}_{\text {st. }}^{2}(0)}{\pi \alpha^{\prime} \tilde{z}_{0}}\left[\int_{C_{0}(\delta) \tilde{z}_{0}^{2}}^{1} \frac{d \xi}{\xi^{2}}\left[\frac{1}{h(\xi)}+\frac{\tilde{\rho}_{\text {st. }}^{2}(0)}{2 h(\xi)^{2}}\right] \sqrt{1+h(\xi) \tilde{\rho}_{\text {st. }}^{\prime 2}(\xi)}\right. \\
& \left.+\int_{C_{0}(\delta) \tilde{z}_{0}^{2}}^{1} \frac{d \xi}{\xi^{2}} \frac{\tilde{\rho}_{\text {st. }}^{\prime}(\xi) \tilde{\delta \rho^{\prime}}(\xi)}{\sqrt{1+h(\xi) \tilde{\rho}_{\text {st. }}^{\prime 2}(\xi)}}\right],
\end{aligned}
$$

which is, to leading order in $\tilde{z}_{0}$,

$$
J \simeq \frac{R^{2} C}{\pi \alpha^{\prime} \tilde{z}_{0}}\left(1-\delta^{4}\right)_{2} F_{1}\left(\frac{1}{2}, \frac{3}{4}, \frac{5}{4}, \delta^{4}\right) .
$$

We interpret the rest mass of a static quark in a thermal medium as the energy of a static string attached to the D7brane at $z=z_{\mathrm{D} 7}$, extending into the black hole horizon at $z=$ $z_{h}$. This energy is found by integrating over the length of the string. Since $z_{h}$ decreases as $T$ increases, bringing the black hole horizon closer to the D7-brane and effectively shortening the string, the energy of the string shrinks with growing $T$. The energy of the static string is given as [87]:

$$
E=-\frac{\sqrt{\lambda}}{2 \pi} \int_{z_{h}}^{z_{D 7}} \frac{d z}{z^{2}}=\frac{\sqrt{\lambda}}{2 \pi}\left(\frac{1}{z_{\mathrm{D} 7}}-\frac{1}{z_{h}}\right) .
$$

When $T=0$, i.e., $z_{h}=\infty$, the energy is simply the quark mass $m_{Q}=\sqrt{\lambda} / 2 \pi z_{\mathrm{D} 7}$. For $T>0$, however, we find the rest mass $M_{\text {rest }}$ to be

$$
M_{\text {rest }}(T)=m_{Q}-\frac{\sqrt{\lambda} T}{2} .
$$

The binding energy of the quarkonia is given by

$$
E_{\text {bind }}=E-2 M_{\text {rest }}(T) \text {. }
$$

Substituting the explicit forms of the dimensionless variables, we can write Eq. (B39) and Eq. (B41) as

$$
\begin{aligned}
E\left(z_{0}\right)= & 2 m_{Q}+\frac{C \sqrt{\lambda}}{2 \pi z_{0}}\left[1-\left(z_{0} \pi T\right)^{4}\right] \\
& \times\left\{3_{2} F_{1}\left[\frac{1}{2}, \frac{3}{4}, \frac{5}{4},\left(z_{0} \pi T\right)^{4}\right]\right. \\
& \left.-4{ }_{2} F_{1}\left[\frac{3}{4}, \frac{3}{2}, \frac{5}{4},\left(z_{0} \pi T\right)^{4}\right]\right\},
\end{aligned}
$$




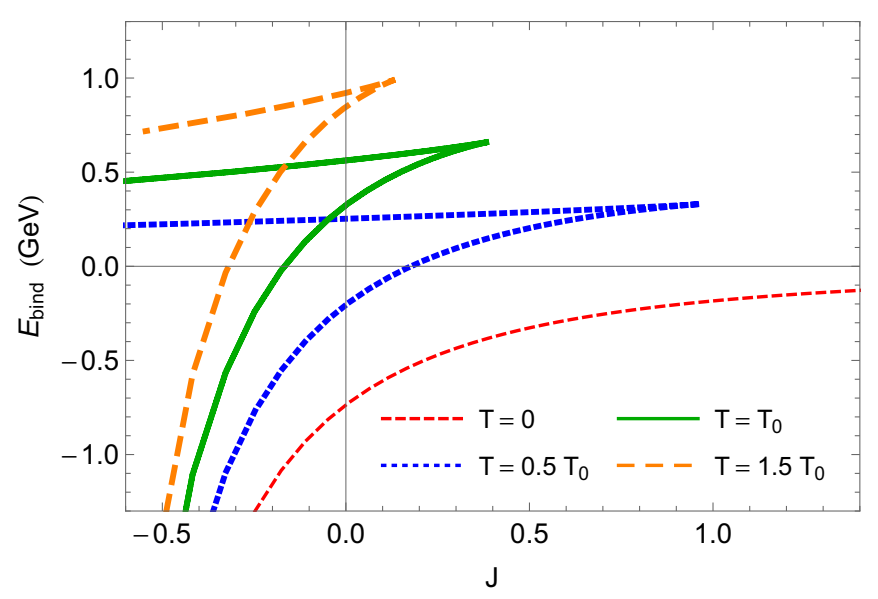

FIG. 7. $E_{\text {bind }}$ for $\Upsilon(1 S)$ as a function of the spin $J$, found solving $E\left(z_{0}\right)$ and $J\left(z_{0}\right)$ from Eqs. (B45) and (B46) parametrically for $z_{0} \in$ $\mathbb{R}$. The dotted blue corresponds to $T=0.5 T_{0}$, solid green to $T=T_{0}$, and the dashed orange to $T=1.5 T_{0}$ for $T_{0}=192 \mathrm{MeV}$. The $E_{\mathrm{bind}}$ for $T=0$ is given as dotted red.

$$
\begin{aligned}
J\left(z_{0}\right)= & \sqrt{\frac{2 C^{3} m_{Q} z_{0}}{\pi}} \lambda^{1 / 4}\left[1-\left(z_{0} \pi T\right)^{4}\right]_{2} F_{1} \\
& \times\left[\frac{1}{2}, \frac{3}{4}, \frac{5}{4},\left(z_{0} \pi T\right)^{4}\right]^{3 / 2} .
\end{aligned}
$$

We note that the expressions for both the energy and spin found for $T>0$ agrees with the results from Kruczenski et al. [73] in the limit $T \rightarrow 0$. In particular, Eqs. (B39) and (B41) agree with Eqs. (4.30) and (4.31) from [73] when taking $T \rightarrow 0$.

When solving parametrically for $E\left(z_{0}\right)$ and $J\left(z_{0}\right)$ from Eqs. (B45) and (B46), one may insist that the string turning point $z_{0}$ be real. To this end, substituting a range of $z_{0} \in \mathbb{R}$ into Eqs. (B45) and (B46) yields binding energies with a hysteresis as shown in Fig. 7. The hysteresis is problematic for two reasons: first, that the spin $J$ has some limit $J^{*}$ is unphysical. Second, the energy is multivalued for $J<J^{*}$.

Therefore, we will require instead that $J$ must be a real parameter, and solve Eq. (B46) for $z_{0}$ which yields a range of $J \in \mathbb{R}$. Doing so yields complex results for $z_{0}$ which can then be substituted into Eq. (B45) for the energy, as shown in Sec. B 3 b.

The real part of $z_{0}$ is shown in Fig. 8(a), and the imaginary part in Fig. 8(b), for $T=T_{0}=192 \mathrm{MeV}$. As was the case in solving for $z_{\max } \in \mathbb{C}$ in Sec. II B, there are two possible roots. For the real part, the solid root yields the physically relevant binding energy i.e., a Coulombic binding energy. For the imaginary part, we choose the root which yields a negative $\operatorname{Im}[E]$, i.e., the solid black line, as the physically relevant one. As argued in Sec. II B, the time evolution of the wave function of any given state is $e^{-i E t} \sim e^{\operatorname{Im}[E] t}$. In order to ensure that the probability of a single state does not exceed one, we require that $\operatorname{Im}[E]<0[61]$.

\section{Finite-mass strong-coupling potential}

We wish to compare the binding energies derived for the semiclassical string setup as given in Sec. B 1 to those found

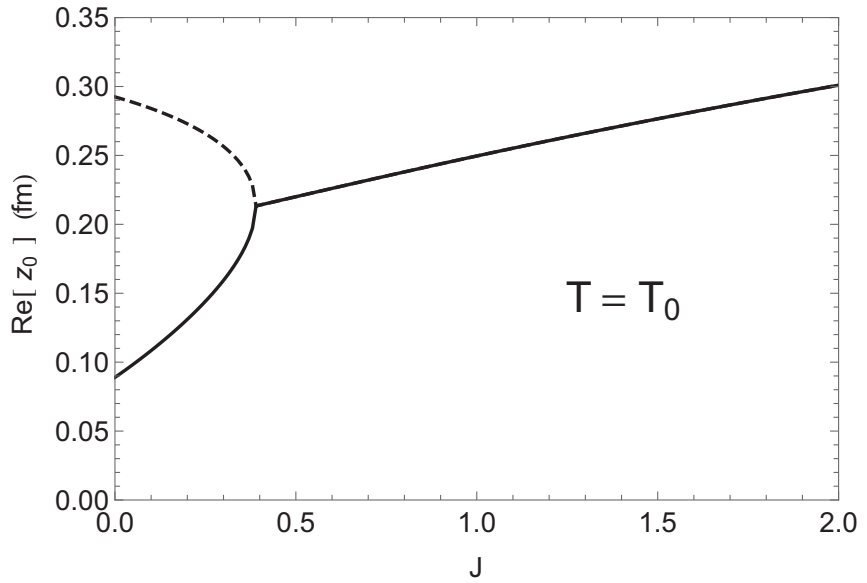

(a)

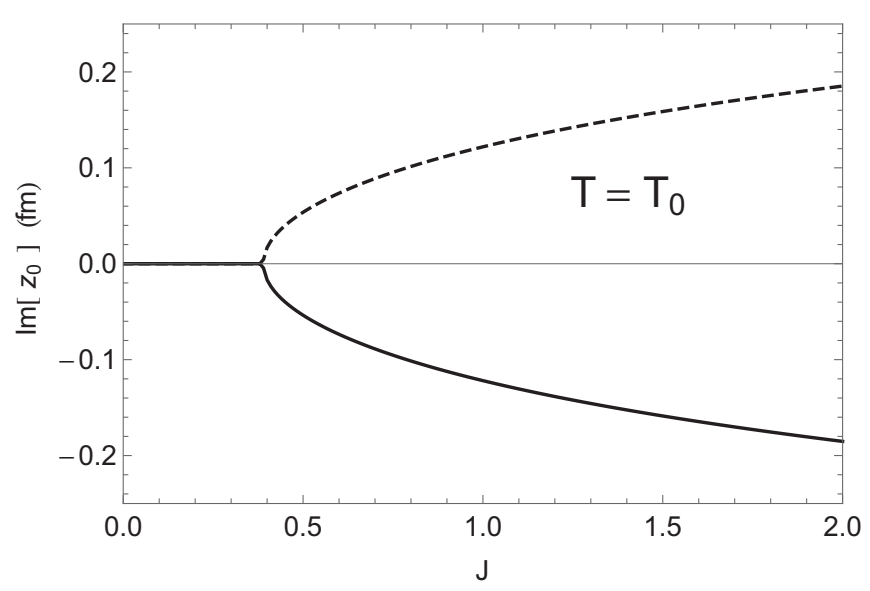

(b)

FIG. 8. All possible solutions for (a) $\operatorname{Re}\left[z_{0}\right]$ and (b) $\operatorname{Im}\left[z_{0}\right]$, from Eq. (B46), as a function of spin $J$ for $T=T_{0}=192 \mathrm{MeV}$. The solid line is the root chosen over the dashed line solution for calculating binding energies.

from the strong-coupling potential given in Sec. II B. A naive comparison of binding energies computed from the strongcoupling potential to those computed in Sec. B 1 led to results which differed even for large $J$, where the binding energies are expected to converge. This discrepancy in binding energies is, however, not surprising, as the strong-coupling potential was derived by Ref. [61] in the infinite-mass limit, whereas the semiclassical string derivation takes the mass to be explicitly finite.

To make an apples-to-apples comparison between the binding energies from the semiclassical string method to those found using the complex strong-coupling potential, then, we generalize the potential given in Sec. II B to finite mass in this section. Following Ref. [61], we consider a semiclassical string at rest, attached to the D7-brane as in Sec. B 1 a. The action is hence as given in Eq. (B10):

$$
S=-\frac{R^{2}}{2 \pi \alpha^{\prime}} \int d t d \sigma \frac{1}{z^{2}} \sqrt{z^{\prime 2}+h(z) \rho_{\mathrm{st}}^{\prime 2}},
$$




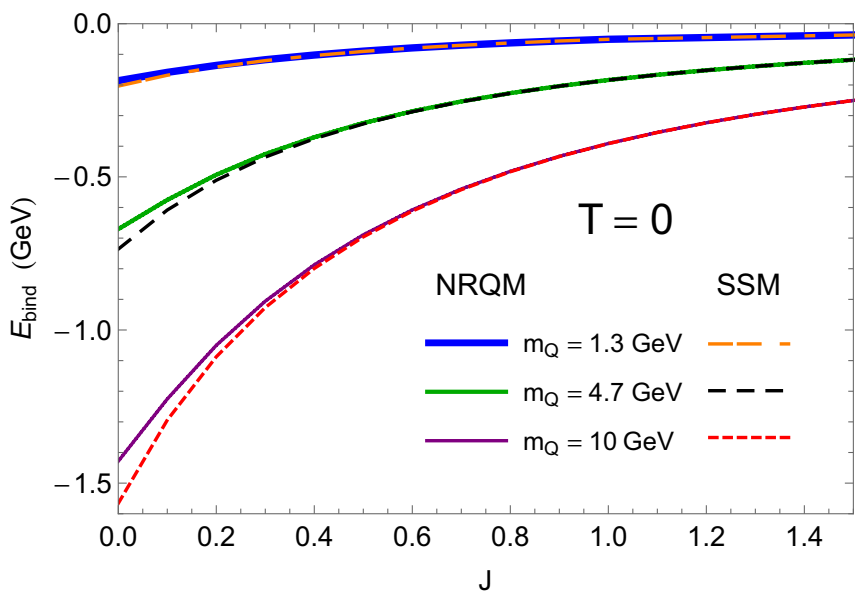

(a)

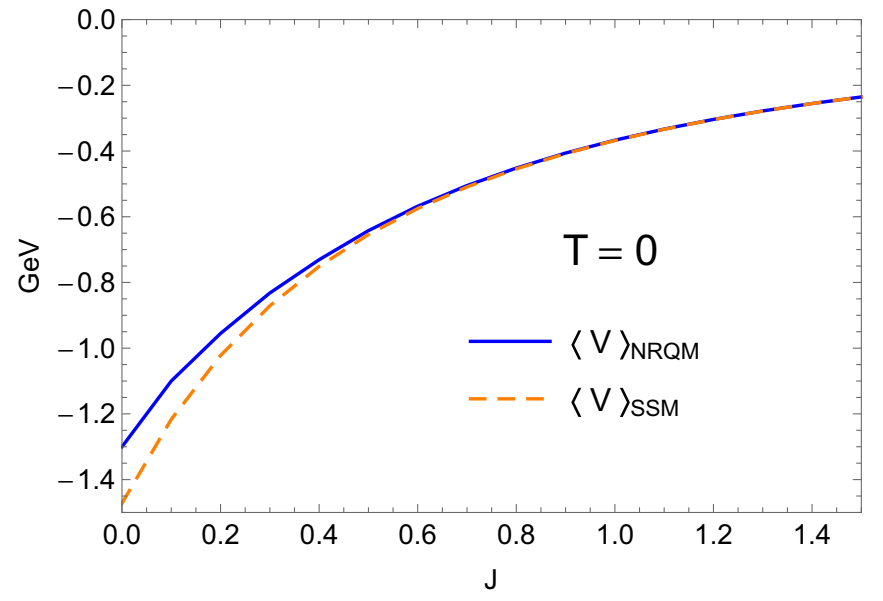

(b)

FIG. 9. (a) The binding energies from NRQM (solid lines) as well as the SSM (dashed lines) for a range of quark masses $m_{Q} \cdot m_{Q}=1.3 \mathrm{GeV}$ is given in thick blue and dashed dotted orange for NRQM and SSM, respectively. Likewise, $m_{Q}=4.7 \mathrm{GeV}$ is given in solid green and dashed black, and $m_{Q}=10 \mathrm{GeV}$ in thin purple and dotted red. (b) The average potential $\langle V\rangle_{\mathrm{NRQM}}$ from NRQM is given in solid blue, and the potential $\langle V\rangle_{\mathrm{SSM}}$ calculated from the SSM is given in dashed orange, for $m_{Q}=4.7 \mathrm{GeV}$.

which was shown to yield Eq. (B16) as solution to its EOM when taking $\sigma \equiv z$ :

$$
\rho_{\text {st. }}^{\prime}(z)=-\frac{z^{2} \sqrt{z_{h}^{4}-z_{0}^{4}}}{\sqrt{\left(z_{h}^{4}-z^{4}\right)\left(z_{0}^{4}-z^{4}\right)}} .
$$

Substituting $h(z)$ and $\rho_{\text {st. }}^{\prime}(z)$, Eq. (B47) becomes

$$
S=-\frac{\sqrt{\lambda}}{\pi} \mathcal{T} \frac{z_{\max }^{2}}{z_{h}^{2}} \int_{z D 7}^{z_{\max }} d z \frac{1}{z^{2}} \sqrt{\frac{z_{h}^{4}-z^{4}}{z_{\max }^{4}-z^{4}}},
$$

where we have defined $\mathcal{T} \equiv \int d t$.

We consider the string endpoints to be attached to the D7brane at $\rho\left(z_{D 7}\right)= \pm r / 2$, where $r$ is the distance between the quark and antiquark in the $q \bar{q}$ pair. Hence from Eq. (B48) we have

$$
\rho\left(z_{D 7}\right)=\int_{z_{D 7}}^{z_{\max }} d x \frac{x^{2} \sqrt{z_{h}^{4}-z_{0}^{4}}}{\sqrt{\left(z_{h}^{4}-x^{4}\right)\left(z_{0}^{4}-x^{4}\right)}},
$$

which yields

$$
\begin{aligned}
\frac{r}{2}= & \sqrt{1-\frac{z_{\max }^{4}}{z_{h}^{4}}}\left[C z_{\max 2} F_{1}\left(\frac{1}{2}, \frac{3}{4}, \frac{5}{4}, \frac{z_{\max }^{4}}{z_{h}^{4}}\right)\right. \\
& \left.-\frac{z_{D 7}^{3}}{3 z_{\max }^{2}} F_{1}\left(\frac{3}{4}, \frac{1}{2}, \frac{1}{2} ; \frac{7}{4} ; \frac{z_{D 7}^{4}}{z_{h}^{4}}, \frac{z_{D 7}^{4}}{z_{\max }^{4}}\right)\right] .
\end{aligned}
$$

$F_{1}$ is the Appell $F_{1}$ function. Equation (B51) can be solved implicitly for $z_{\max }(r)$.

To find the potential, we use [61]

$$
V(r)=-\frac{\left\{S_{\mathrm{NG}}(r, T)-\operatorname{Re}\left[S_{\mathrm{NG}}(\infty, T)\right]\right\}}{\mathcal{T}} .
$$

$S_{\mathrm{NG}}(\infty, T)$ is the action of two straight hanging strings attached to the D7-brane at $\rho\left(z_{D 7}\right)= \pm r / 2$ :

$$
S_{\mathrm{NG}}(\infty, T)=-\frac{\sqrt{\lambda}}{\pi} \mathcal{T} \int_{z_{D 7}}^{z_{h}} \frac{d z}{z^{2}}
$$

Hence the finite-mass potential from Eq. (B52) is

$$
\begin{aligned}
V(r)= & -\frac{\sqrt{\lambda}}{\pi z_{\max }}\left\{C_{2} F_{1}\left(-\frac{1}{2},-\frac{1}{4}, \frac{1}{4}, \frac{z_{\max }^{4}}{z_{h}^{4}}\right)-\frac{z_{\max }}{z_{h}}\right. \\
& \left.+\frac{z_{\max }}{z_{D 7}}\left[1-F_{1}\left(-\frac{1}{4},-\frac{1}{2}, \frac{1}{2}, \frac{3}{4}, \frac{z_{D 7}^{4}}{z_{h}^{4}}, \frac{z_{D 7}^{4}}{z_{\max }^{4}}\right)\right]\right\} .
\end{aligned}
$$

In order to compare binding energies computed from the above potential using the imaginary time method given in Sec. III, we need to extend Sec. III to consider $J>0$. To this end, we modify the Hamiltonian given in Eq. (9) as follows:

$$
H=-\frac{1}{2 m}\left[\nabla^{2}-\frac{J(J+1)}{r^{2}}\right]+V(r) .
$$

All then remains the same, barring $W(\rho)$ given in Eq. (12), which becomes

$$
W(\rho) \equiv \frac{J(J+1)}{2 \rho^{2}}+\frac{V(\rho)}{m} .
$$

\section{Comparison of binding energies}

$$
\text { a. } T=0
$$

For the simplest $T=0$ case, both the binding energies from the SSM given in Sec. B 1, and the imaginary time numerical method (NRQM) given in Sec. B 2, are purely real. These binding energies are shown in Fig. 9(a) for a range of quark masses. Clearly the binding energies from the two methodologies agree for large $J$. At small $J$, however, $E_{\text {bind }}^{\mathrm{NRQM}}>E_{\text {bind }}^{\mathrm{SSM}}$. 


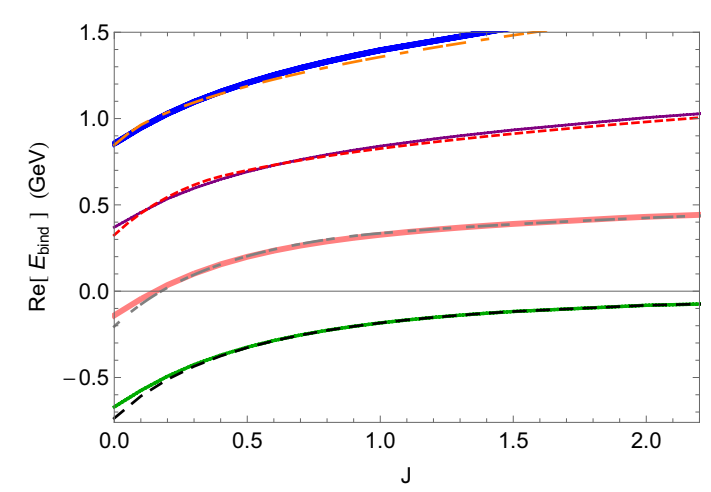

(a)

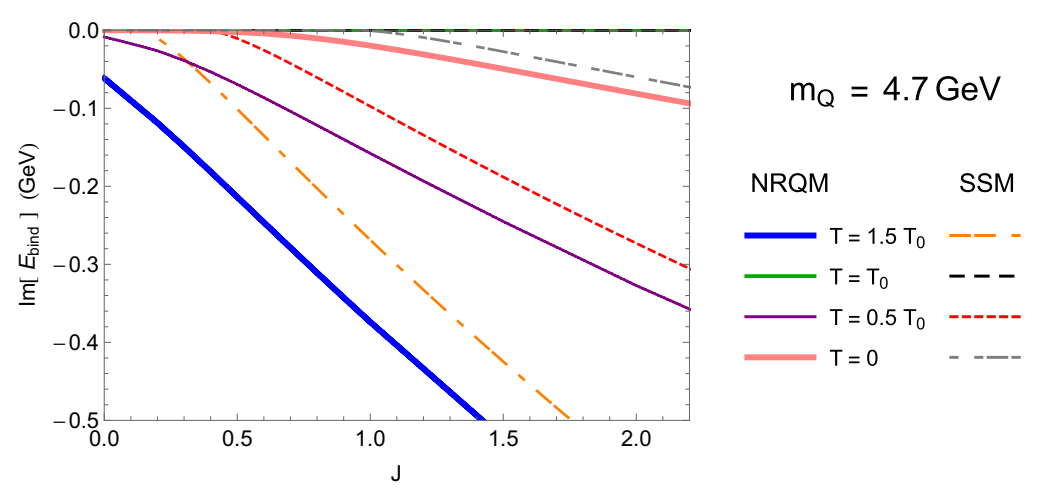

(b)

FIG. 10. The (a) real and (b) imaginary parts of the binding energies of $\Upsilon\left(1 \mathrm{~S}\right.$ ) for a range of temperatures $T . T=1.5 T_{0}$ is given in thick blue and dashed dotted orange for NRQM and SSM, respectively. Likewise, $T=T_{0}$ is given in thin purple and dotted red, and $T=0.5 T_{0}$ in thick pink and dashed dotted gray. $T=0$ is included in solid green and dashed black.

This discrepancy occurs since the imaginary time numerical method takes into account quantum effects, whereas the SSM does not. The wave functions are more spread out when considering quantum mechanics, leading to less tightly bound quarkonia.

We may explicitly demonstrate the effect of this quantum spreading by investigating the potential probed by the two methods. We show in Fig. 9(b) the average potential felt by the heavy quarks in the two methods. The average potential $\langle V\rangle_{\mathrm{NRQM}}$ from NRQM is simply found numerically using:

$$
\langle V\rangle_{\mathrm{NRQM}}=\frac{\int r^{2} d r \psi_{J}(r)^{*} V(r) \psi_{J}(r)}{\int r^{2} d r\left|\psi_{J}(r)\right|^{2}}
$$

We calculate the potential $\langle V\rangle_{\text {SSM }}$ from the SSM using that $E=\omega J-L=T+V \Rightarrow T=\frac{1}{2} \omega J$. From Ref. [73] we have for $T=0$ :

$$
\omega \simeq \frac{C \tilde{z}_{0}^{3}}{z_{D 7}} \quad \text { and } \quad \tilde{z}_{0}=\frac{\sqrt{\lambda} C}{\pi J} .
$$

Hence we can write

$$
T(J)=\frac{\lambda C^{4}}{\pi^{2} J^{2}} m_{Q}
$$

and $\langle V\rangle_{\mathrm{SSM}}=E(J)-T(J)$ follows.

It is evident from Fig. 9(b) that $\langle V\rangle_{\mathrm{NRQM}}>\langle V\rangle_{\mathrm{SSM}}$ : The average potential experienced by the heavy quarks is deeper at small $J$ for the SSM as opposed to NRQM. We may under- stand the ordering of potentials from the quantum spreading the NRQM wave function preferentially explores the potential at larger $r$ due to uncertainty. As a result, the binding energies are therefore less negative when quantum effects are taken into account, i.e., the quarkonia is less strongly bound, as expected.

\section{b. $T>0$}

Figures 10(a) and 10(b) show the real and imaginary parts, respectively, of the binding energies from NRQM and the SSM for $\Upsilon(1 \mathrm{~S})$ for a range of temperatures.

Consider first the real part. At small $J$, the real part of the NRQM binding energy is slightly larger than the real part of the binding energy from the SSM, as was the case for $T=0$.

The imaginary part of the binding energies echo the conclusions drawn regarding the difference in the real parts. The binding energies from NRQM become complex at smaller $J$ than those from the SSM. The larger the imaginary part of the binding energy, the greater the tendency for the quarkonia to fall apart: The $\Upsilon(1 \mathrm{~S})$ in the NRQM picture is again less tightly bound than SSM.

For large $J$, however, the binding energies converge as expected. The binding energies differ slightly only for small $J$, a discrepancy accounted for by quantum mechanics. The excellent agreement of these binding energies shows that the use of complex heavy quark potentials to compute binding energies is consistent with the independent semiclassical string method.
[1] J. Adams et al. (STAR Collaboration), Experimental and theoretical challenges in the search for the quark gluon plasma: The STAR Collaboration's critical assessment of the evidence from RHIC collisions, Nucl. Phys. A 757, 102 (2005).

[2] K. Adcox et al. (PHENIX Collaboration), Formation of dense partonic matter in relativistic nucleus-nucleus collisions at RHIC: Experimental evaluation by the PHENIX collaboration, Nucl. Phys. A 757, 184 (2005).

[3] Georges Aad et al. (ATLAS Collaboration), Observation of a Centrality-Dependent Dijet Asymmetry in Lead-Lead Col- lisions at $\sqrt{s_{N N}}=2.77 \mathrm{TeV}$ with the ATLAS Detector at the LHC, Phys. Rev. Lett. 105, 252303 (2010).

[4] V. Khachatryan et al. (CMS Collaboration), Observation of long-range near-side angular correlations in proton-proton collisions at the LHC, J. High Energy Phys. 09 (2010) 091.

[5] G. Aad et al. (ATLAS Collaboration), Observation of Associated Near-Side and Away-Side Long-Range Dorrelations in $\sqrt{s_{N N}}=5.02 \mathrm{TeV}$ Proton-Lead Collisions with the ATLAS Detector, Phys. Rev. Lett. 110, 182302 (2013). 
[6] B. Abelev et al. (ALICE Collaboration), Long-range angular correlations on the near and away side in $p$ - $\mathrm{Pb}$ collisions at $\sqrt{s_{N N}}=5.02 \mathrm{TeV}$, Phys. Lett. B 719, 29 (2013).

[7] S. Chatrchyan et al. (CMS Collaboration), Observation of Sequential Upsilon Suppression in $\mathrm{PbPb}$ Collisions, Phys. Rev. Lett. 109, 222301 (2012).

[8] M. Gyulassy and L. McLerran, New forms of QCD matter discovered at RHIC, Nucl. Phys. A 750, 30 (2005).

[9] D. Teaney, The Effects of viscosity on spectra, elliptic flow, and HBT radii, Phys. Rev. C 68, 034913 (2003).

[10] P. M. Chesler and L. G. Yaffe, Holography and Colliding Gravitational Shock Waves in Asymptotically $\mathrm{AdS}_{5}$ Spacetime, Phys. Rev. Lett. 106, 021601 (2011).

[11] H. Song, S. A. Bass, U. Heinz, T. Hirano, and C. Shen, 200 A $\mathrm{GeV} \mathrm{Au}+\mathrm{Au}$ Collisions Serve a Nearly Perfect Quark-Gluon Liquid, Phys. Rev. Lett. 106, 192301 (2011).

[12] C. Gale, S. Jeon, B. Schenke, P. Tribedy, and R. Venugopalan, Event-By-Event Anisotropic Flow in Heavy-Ion Collisions from Combined Yang-Mills and Viscous Fluid Dynamics, Phys. Rev. Lett. 110, 012302 (2013).

[13] J. E. Bernhard, J. S. Moreland, S. A. Bass, J. Liu, and U. Heinz, Applying Bayesian parameter estimation to relativistic heavyion collisions: Simultaneous characterization of the initial state and quark-gluon plasma medium, Phys. Rev. C 94, 024907 (2016).

[14] M. Alqahtani, M. Nopoush, R. Ryblewski, and M. Strickland, Anisotropic hydrodynamic modeling of $2.76 \mathrm{TeV} \mathrm{Pb}-\mathrm{Pb}$ collisions, Phys. Rev. C 96, 044910 (2017).

[15] R. Morad and W. A. Horowitz, Strong-coupling jet energy loss from AdS/CFT, J. High Energy Phys. 11 (2014) 017.

[16] W. A. Horowitz, Fluctuating heavy quark energy loss in a strongly coupled quark-gluon plasma, Phys. Rev. D 91, 085019 (2015).

[17] R. Hambrock and W. A. Horowitz, AdS/CFT predictions for azimuthal and momentum correlations of $b \bar{b}$ pairs in heavy ion collisions, Nucl. Part. Phys. Proc. 289, 233 (2017).

[18] J. Brewer, K. Rajagopal, A. Sadofyev, and W. van der Schee, Holographic jet shapes and their evolution in strongly coupled plasma, Nucl. Phys. A 967, 508 (2017).

[19] R. D. Weller and P. Romatschke, One fluid to rule them all: Viscous hydrodynamic description of event-by-event central $\mathrm{p}+\mathrm{p}, \mathrm{p}+\mathrm{Pb}$ and $\mathrm{Pb}+\mathrm{Pb}$ collisions at $\sqrt{s}=5.02 \mathrm{TeV}$, Phys. Lett. B 774, 351 (2017).

[20] D. Molnar and M. Gyulassy, Saturation of elliptic flow and the transport opacity of the gluon plasma at RHIC, Nucl. Phys. A 697, 495 (2002).

[21] Zi-wei Lin and C. M. Ko, Partonic effects on the elliptic flow at RHIC, Phys. Rev. C 65, 034904 (2002).

[22] A. Bzdak and Guo-Liang Ma, Elliptic and Triangular Flow in $p+\mathrm{Pb}$ and Peripheral $\mathrm{Pb}+\mathrm{Pb}$ Collisions from Parton Scatterings, Phys. Rev. Lett. 113, 252301 (2014).

[23] J. D. Orjuela Koop, A. Adare, D. McGlinchey, and J. L. Nagle, Azimuthal anisotropy relative to the participant plane from a multiphase transport model in central $\mathrm{p}+\mathrm{Au}, \mathrm{d}+\mathrm{Au}$, and ${ }^{3} \mathrm{He}+$ $\mathrm{Au}$ collisions at $\sqrt{s_{N N}}=200 \mathrm{GeV}$, Phys. Rev. C 92, 054903 (2015).

[24] L. He, T. Edmonds, Z.-W. Lin, F. Liu, D. Molnar, and F. Wang, Anisotropic parton escape is the dominant source of azimuthal anisotropy in transport models, Phys. Lett. B 753, 506 (2016).
[25] Z.-W. Lin, L. He, T. Edmonds, F. Liu, D. Molnar, and F. Wang, Elliptic anisotropy $v_{2}$ may be dominated by particle escape instead of hydrodynamic flow, Nucl. Phys. A 956, 316 (2016).

[26] M. Gyulassy, P. Levai, and I. Vitev, Jet tomography of Au+Au reactions including multigluon fluctuations, Phys. Lett. B 538, 282 (2002).

[27] I. Vitev and M. Gyulassy, High $p_{T}$ Tomography of $d+\mathrm{Au}$ and $\mathrm{Au}+\mathrm{Au}$ at SPS, RHIC, and LHC, Phys. Rev. Lett. 89, 252301 (2002).

[28] E. Wang and X.-N. Wang, Jet Tomography of Dense and Nuclear Matter, Phys. Rev. Lett. 89, 162301 (2002).

[29] A. Majumder, E. Wang, and X.-N. Wang, Modified Dihadron Fragmentation Functions in hot and Nuclear Matter, Phys. Rev. Lett. 99, 152301 (2007).

[30] A. Dainese, C. Loizides, and G. Paic, Leading-particle suppression in high energy nucleus-nucleus collisions, Eur. Phys. J. C 38, 461 (2005).

[31] N. Armesto, M. Cacciari, A. Dainese, C. A. Salgado, and U. A. Wiedemann, How sensitive are high- $\mathrm{p}(\mathrm{T})$ electron spectra at RHIC to heavy quark energy loss? Phys. Lett. B 637, 362 (2006).

[32] S. Wicks, W. Horowitz, M. Djordjevic, and M. Gyulassy, Elastic, inelastic, and path length fluctuations in jet tomography, Nucl. Phys. A 784, 426 (2007).

[33] A. Majumder, C. Nonaka, and S. A. Bass, Jet modification in three dimensional fluid dynamics at next-to-leading twist, Phys. Rev. C 76, 041902(R) (2007).

[34] H. Zhang, J. F. Owens, E. Wang, and X.-N. Wang, Tomography of High-Energy Nuclear Collisions with Photon-Hadron Correlations, Phys. Rev. Lett. 103, 032302 (2009).

[35] I. Vitev and B.-W. Zhang, Jet Tomography of High-Energy Nucleus-Nucleus Collisions at Next-To-Leading Order, Phys. Rev. Lett. 104, 132001 (2010).

[36] B. Schenke, C. Gale, and S. Jeon, MARTINI: An Event generator for relativistic heavy-ion collisions, Phys. Rev. C 80, 054913 (2009).

[37] C. Young, B. Schenke, S. Jeon, and C. Gale, Dijet asymmetry at the energies available at the CERN Large Hadron Collider, Phys. Rev. C 84, 024907 (2011).

[38] A. Majumder and C. Shen, Suppression of the High $p_{T}$ Charged Hadron $R_{A A}$ at the LHC, Phys. Rev. Lett. 109, 202301 (2012).

[39] W. A. Horowitz and M. Gyulassy, The surprising transparency of the sQGP at LHC, Nucl. Phys. A 872, 265 (2011).

[40] A. Buzzatti and M. Gyulassy, Jet Flavor Tomography of Quark Gluon Plasmas at RHIC and LHC, Phys. Rev. Lett. 108, 022301 (2012).

[41] W. A. Horowitz, Heavy quark production and energy loss, Nucl. Phys. A 904-905, 186c (2013).

[42] M. Djordjevic and M. Djordjevic, LHC jet suppression of light and heavy flavor observables, Phys. Lett. B 734, 286 (2014).

[43] H. Liu, K. Rajagopal, and U. A. Wiedemann, Calculating the Jet Quenching Parameter from AdS/CFT, Phys. Rev. Lett. 97, 182301 (2006).

[44] J. Casalderrey-Solana, D. C. Gulhan, J. G. Milhano, D. Pablos, and K. Rajagopal, A hybrid strong/weak coupling approach to jet quenching, J. High Energy Phys. 10 (2014) 019. 
[45] J. Casalderrey-Solana, D. C. Gulhan, J. G. Milhano, D. Pablos, and K. Rajagopal, Predictions for boson-jet observables and fragmentation function ratios from a hybrid strong/weak coupling model for jet quenching, J. High Energy Phys. 03 (2016) 053.

[46] J. Casalderrey-Solana, D. Gulhan, G. Milhano, D. Pablos, and K. Rajagopal, Angular structure of jet quenching within a hybrid strong/weak coupling model, J. High Energy Phys. 03 (2017) 135.

[47] C. Patrignani et al. (Particle Data Group), Review of particle physics, Chin. Phys. C 40, 100001 (2016).

[48] T. Matsui and H. Satz, $J / \psi$ suppression by quark-gluon plasma formation, Phys. Lett. B 178, 416 (1986).

[49] F. Karsch, M. T. Mehr, and H. Satz, Color screening and deconfinement for bound states of heavy quarks, Z. Phys. C 37, 617 (1988).

[50] F. Karsch, D. Kharzeev, and H. Satz, Sequential charmonium dissociation, Phys. Lett. B 637, 75 (2006).

[51] M. Laine, O. Philipsen, P. Romatschke, and M. Tassler, Realtime static potential in hot QCD, J. High Energy Phys. 03 (2007) 054

[52] A. Beraudo, J. P. Blaizot, and C. Ratti, Real and imaginary-time $\mathrm{Q}$ anti-Q correlators in a thermal medium, Nucl. Phys. A $\mathbf{8 0 6}$, 312 (2008).

[53] Y. Burnier, M. Laine, and M. Vepsalainen, Heavy quarkonium in any channel in resummed hot QCD, J. High Energy Phys. 01 (2008) 043.

[54] M. Laine, O. Philipsen, and M. Tassler, Thermal imaginary part of a real-time static potential from classical lattice gauge theory simulations, J. High Energy Phys. 09 (2007) 066.

[55] A. Rothkopf, T. Hatsuda, and S. Sasaki, Complex Heavy-Quark Potential at Finite Temperature from Lattice QCD, Phys. Rev. Lett. 108, 162001 (2012).

[56] N. Brambilla, J. Ghiglieri, A. Vairo, and P. Petreczky, Static quark-antiquark pairs at finite temperature, Phys. Rev. D 78, 014017 (2008).

[57] A. Andronic et al., Heavy-flavour and quarkonium production in the LHC era: From proton-proton to heavy-ion collisions, Eur. Phys. J. C 76, 107 (2016).

[58] G. Aarts et al., Heavy-flavor production and medium properties in high-energy nuclear collisions - What next? Eur. Phys. J. A 53, 93 (2017).

[59] S.-J. Rey, S. Theisen, and J.-T. Yee, Wilson-Polyakov loop at finite temperature in large $\mathrm{N}$ gauge theory and anti-de Sitter supergravity, Nucl. Phys. B 527, 171 (1998).

[60] A. Brandhuber, N. Itzhaki, J. Sonnenschein, and S. Yankielowicz, Wilson loops in the large $\mathrm{N}$ limit at finite temperature, Phys. Lett. B 434, 36 (1998).

[61] J. L. Albacete, Y. V. Kovchegov, and A. Taliotis, Heavy quark potential at finite temperature in AdS/CFT revisited, Phys. Rev. D 78, 115007 (2008).

[62] J. Noronha and A. Dumitru, Thermal Width of the $\Upsilon$ at Large 't Hooft Coupling, Phys. Rev. Lett. 103, 152304 (2009).

[63] T. Hayata, K. Nawa, and T. Hatsuda, Time-dependent heavyquark potential at finite temperature from gauge-gravity duality, Phys. Rev. D 87, 101901(R) (2013).
[64] K. Bitaghsir Fadafan and S. K. Tabatabaei, The imaginary potential and thermal width of moving quarkonium from holography, J. Phys. G 43, 095001 (2016).

[65] H. Liu, K. Rajagopal, and U. A. Wiedemann, An AdS/CFT Calculation of Screening in a Hot Wind, Phys. Rev. Lett. 98, 182301 (2007).

[66] S. I. Finazzo and J. Noronha, Thermal suppression of moving heavy quark pairs in a strongly coupled plasma, J. High Energy Phys. 01 (2015) 051.

[67] N. R. F. Braga and L. F. Ferreira, Thermal width of heavy quarkonia from an AdS/QCD model, Phys. Rev. D 94, 094019 (2016).

[68] B. K. Patra, H. Khanchandani, and L. Thakur, Velocity-induced heavy quarkonium dissociation using the gauge-gravity correspondence, Phys. Rev. D 92, 085034 (2015).

[69] M. Ali-Akbari, D. Giataganas, and Z. Rezaei, Imaginary potential of heavy quarkonia moving in strongly coupled plasma, Phys. Rev. D 90, 086001 (2014).

[70] M. A. Escobedo, F. Giannuzzi, M. Mannarelli, and J. Soto, Heavy quarkonium moving in a quark-gluon plasma, Phys. Rev. D 87, 114005 (2013).

[71] B. Krouppa, R. Ryblewski, and M. Strickland, Bottomonia suppression in 2.76 TeV Pb-Pb collisions, Phys. Rev. C 92, 061901(R) (2015).

[72] D. Kraft, Stochastic Variational Approaches to Non-Hermitian Quantum-Mechanical Problems, Master's thesis, Graz University (2013).

[73] M. Kruczenski, D. Mateos, R. C. Myers, and D. J. Winters, Meson spectroscopy in AdS / CFT with flavor, J. High Energy Phys. 07 (2003) 049.

[74] M. Strickland and D. Bazow, Thermal bottomonium suppression at RHIC and LHC, Nucl. Phys. A 879, 25 (2012).

[75] A. Dumitru, Y. Guo, and M. Strickland, The Imaginary part of the static gluon propagator in an anisotropic (viscous) QCD plasma, Phys. Rev. D 79, 114003 (2009).

[76] O. Kaczmarek, F. Karsch, F. Zantow, and P. Petreczky, Static quark anti-quark free energy and the running coupling at finite temperature, Phys. Rev. D 70, 074505 (2004).

[77] J. M. Maldacena, Wilson Loops in Large N Field Theories, Phys. Rev. Lett. 80, 4859 (1998).

[78] C. Ewerz, O. Kaczmarek, and A. Samberg, Free energy of a heavy quark-antiquark pair in a thermal medium from AdS/CFT J. High Energy Phys. 03 (2018) 088.

[79] M. Margotta, K. McCarty, C. McGahan, M. Strickland, and D. Yager-Elorriaga, Quarkonium states in a complex-valued potential, Phys. Rev. D 83, 105019 (2011).

[80] I. W. Sudiarta and D. J. W. Geldart, Solving the schrödinger equation using the finite difference time domain method, J. Phys. A: Math. Theor. 40, 1885 (2007).

[81] W. H. Press, S. A. Teukolsky, W. T. Vetterling, and B. P. Flannery, Numerical Recipes 3rd Edition: The Art of Scientific Computing, 3rd ed. (Cambridge University Press, New York, 2007), pp. 1043-1046.

[82] M. L. Miller, K. Reygers, S. J. Sanders, and P. Steinberg, Glauber modeling in high energy nuclear collisions, Ann. Rev. Nucl. Part. Sci. 57, 205 (2007). 
[83] V. Khachatryan et al. (CMS Collaboration), Suppression of $\Upsilon(1 S), \Upsilon(2 S)$ and $\Upsilon(3 S)$ production in $\mathrm{PbPb}$ collisions at $\sqrt{s_{\mathrm{NN}}}=2.76 \mathrm{TeV}$, Phys. Lett. B 770, 357 (2017).

[84] S. S. Gubser, Momentum fluctuations of heavy quarks in the gauge-string duality, Nucl. Phys. B 790, 175 (2008).

[85] A. Messiah, Quantum Mechanics (Dover, London, 2014).
[86] S. S. Gubser, Drag force in AdS/CFT, Phys. Rev. D 74, 126005 (2006).

[87] C. P. Herzog, A. Karch, P. Kovtun, C. Kozcaz, and L. G. Yaffe, Energy loss of a heavy quark moving through $\mathrm{N}=4$ supersymmetric Yang-Mills plasma, J. High Energy Phys. 07 (2006) 013. 